\title{
Wigner and Racah coefficients for $\mathrm{SU}_{3}{ }^{*}$
}

\section{J. P. Draayer ${ }^{\dagger}$ and Yoshimi Akiyama $\ddagger$}

Department of Physics, The University of Michigan, Ann Arbor, Michigan 48105

(Received 19 March 1973; revised manuscript received 22 May 1973)

A general yet simple and hence practical algorithm for calculating $S U_{3} \supset S U_{2} \times U_{1}$ Wigner coefficients is formulated. The resolution of the outer multiplicity follows the prescription given by Biedenharn and Louck. It is shown that $\mathrm{SU}_{3}$ Racah coefficients can be obtained as a solution to a set of simultaneous equations with unknown coefficients given as a by-product of the initial steps in the $\mathrm{SU}_{3} \supset \mathrm{SU}_{2} \times U_{1}$ Wigner coefficient construction algorithm. A general expression for evaluating $S U_{3} \supset R_{3}$ Wigner coefficients as a sum over a simple subset of the corresponding $S U_{3} \supset S U_{2} \times U_{1}$ Wigner coefficients is also presented. State conjugation properties are discussed and symmetry relations for both the $S U_{3} \supset S U_{2} \times U_{1}$ and $S U_{3} \supset R_{3}$ Wigner coefficients are given. Machine codes based on the results are available.

\section{INTRODUCTION}

The work of Wigner on the theory of group representations ${ }^{1}$ coupled with Racah's development of the algebra of tensor operator $\mathrm{s}^{2}$ provides basic simplifying techniques for spectroscopic analyses. The usefulness of their techniques in any particular situation, however, depends to a great extent upon the availability of the appropriate Wigner and Racah coefficients. Ordinary angular momentum algebra, for example, owes its utility as a calculational tool to the ready availability of $S U_{2}$ Wigner and Racah coefficients. Other more complicated group structures for which Wigner and Racah coefficients are not so readily available, however, are also known to have real physical significance. The special unitary group in three dimensions, $S U_{3}$, is a case in point. In 1958 Elliott pointed out its usefulness in understanding the rotational structure of light nuclei. ${ }^{3}$ Some four years later it was also recognized as being of importance in the classification of elementary particles. ${ }^{4}$ As a consequence, Wigner and Racah coefficients for this group have been given in either algebraic or numeric form for simple cases of special interest by a number of authors. 5 More general results have only recently been made available through the work of Biedenharn and Louck and co-workers. ${ }^{6-12}$ Except for the case of multiplicity free and the so-called $\Gamma_{s}$ couplings, however, an additional algorithm is needed if numerical values for Wigner coefficients are to be extracted from the formalism. And since most authors disagree in their choice of a phase convention, extreme caution must be used if results so obtained are used to augment simple algebraic formulas currently available. An additional complication exists because two inequivalent reductions are needed: $S U_{3} \supset$ $S U_{2} \times U_{1}$ in particle physics and $S U_{3} \supset R_{3}$ in nuclear physics.

The purpose of this article is to: (i) Formulate in the spirit of an ordinary tensor formalism (built with tensors which by construction have the same null space properties as the Biedenharn and Louck Wigner operators) a general but simple and hence practical algorithm for generating $\mathrm{SU}_{3} \supset \mathrm{SU}_{2} \times U_{1}$ Wigner coefficients for arbitrary couplings and multiplicities; (ii) express $S U_{3}$ Racah coefficients as the solution to a set of simultaneous equations with the unknown coefficients given as a by-product of the initial steps in the $S U_{3} \supset S U_{2} \times U_{1}$ Wigner coefficient construction algorithm; (iii) exploit properties of the $S U_{3} \supset R_{3}$ projection process together with known transformation coefficients between the $S U_{3}$ $\supset S U_{2} \times U_{1}$ and $S U_{3} \supset R_{3}$ schemes to express $S U_{3} \supset R_{3}$ Wigner coefficients as a sum over a particularly simple subset of the corresponding $S U_{3} \supset S U_{2} \times U_{1}$ Wigner coefficients; (iv) list symmetry properties of the transformation coefficients between the $S U_{3} \supset S U_{2} \times U_{1}$ and
$S U_{3} \supset R_{3}$ schemes and discuss conjugation properties of state vectors for both reductions; ( $v$ ) give symmetry properties for both the $S U_{3} \supset S U_{2} \times U_{1}$ and $S U_{3} \supset R_{3}$ Wigner coefficients. We begin by briefly reviewing common notations and discussing their relationship to one another.

\section{BASIC NOTATION}

The labels $\lambda$ and $\mu$ are used to characterize the irreducible representations of $S U_{3}$. The row labels in the $S U_{3} \supset S U_{2} \times U_{1}$ reduction are chosen as

$$
\begin{aligned}
& \epsilon=2 \lambda+\mu-3(p+q)=-3 Y, \\
& \Lambda=(\mu+p-q) / 2=I, \\
& M_{\Lambda}=r-\Lambda=I_{z},
\end{aligned}
$$

where the integers $p, q, r$ satisfy $0 \leq p \leq \lambda, 0 \leq q \leq \mu$, $0 \leq r \leq 2 \Lambda$. The notation $\left|(\lambda \mu) \in \Lambda M_{\Lambda}\right\rangle$ is that introduced by Elliott into nuclear physics to label states in the socalled intrinsic or body-fixed system. ${ }^{3,13}$ In terms of a three-dimensional oscillator with $n_{i}$ quanta in the $i$ direction, $\epsilon=2 n_{3}-n_{1}-n_{2}$ while $\Lambda$ labels the irreducible representation of $S U_{2}$ with projection $M_{\Lambda}=\left(n_{1}-n_{2}\right) / 2$. In particle physics states are labeled as $\left|(\lambda \mu) Y I I_{z}\right\rangle$ with $Y$ denoting the hypercharge and $I$ and $I_{z}$ the isospin and its projection, respectively. ${ }^{14}$

An equivalent but mathematically more elegant notation is that due to Gel'fand in which case states are labeled by patterns of the type 15

$$
|G\rangle \equiv\left|\begin{array}{ccc}
g_{13} & g_{23} & g_{33} \\
& g_{12} & g_{22} \\
& g_{11} &
\end{array}\right|
$$

The $g_{i j}, 1 \leq i \leq j \leq 3$, specify the irreducible representation of $U_{j}$ in the chain $U_{3} \supset U_{2} \supset U_{1}$. Specifically, $g_{i j}$ is the number of boxes in row $i$ of the Young tableau for $U_{j} . \lambda=g_{13}-g_{23}, \mu=g_{23}-g_{33}$, and $\nu=g_{33}$ are then the number of columns containing 1,2 , and 3 boxes, respectively, in the Young tableau for $U_{3}$. For notational convenience $G$ (for Gel'fand) will be used to denote the full set of $g_{i j}$ labels. Apart from an $n_{i}$-dependent phase factor $|G\rangle=\left|(\lambda \mu) \in \Lambda M_{\Lambda}\right\rangle$ with $g_{12}=p+\mu+\nu=\frac{1}{3}(\lambda+$ $2 \mu)-\frac{1}{6} \epsilon+\Lambda+\nu, g_{22}=q+\nu=\frac{1}{3}(\lambda+2 \mu)-\frac{1}{6} \epsilon-\Lambda+$ $\nu, g_{11}=r+q+\nu=2 M_{\Lambda}+\frac{1}{3}(\lambda+2 \mu)-\frac{1}{6} \epsilon-3 \Lambda+\nu$. The so-called betweenness conditions $\left(g_{i j} \geq g_{i, j-1} \geq\right.$ $\left.g_{i+1, j}\right)$ are equivalent to the restrictions $0 \leq p \leq \lambda, 0 \leq$ $q \leq \mu, 0 \leq r \leq 2 \Lambda$.

States of particular interest are those for which the number of oscillator quanta $\left(n_{i}=\sum_{j} g_{j, i}-\sum_{j} g_{j, i-1}\right)$ in 
TABLE 1. (Subgroup labels for extremal states). The subscripts HW and LW mean highest weight and lowest weight in the Gel'fand sense (not to be confused with $\epsilon_{\max } \sim G_{\mathrm{LW}}$ and $\epsilon_{\mathrm{min}} \sim G_{\mathrm{HW}}$ ).

\begin{tabular}{|c|c|c|c|c|c|c|c|c|c|c|c|c|c|c|}
\hline $\bar{G}$ & $g_{12}$ & $\overline{g_{22}}$ & $g_{11}$ & $\bar{p}$ & $q$ & $r$ & $\vec{\epsilon}$ & $2 \Lambda$ & $2 M_{\Lambda}$ & $n_{1}$ & $n_{2}$ & $n_{3}$ & $I$ & $\bar{J}$ \\
\hline$G_{H W}$ & $g_{13}$ & $g_{23}$ & $g_{13}$ & $\lambda$ & $\mu$ & $\lambda$ & $-\lambda-2 \mu$ & $\lambda$ & $\lambda$ & $\lambda+\mu+\nu$ & $\mu+\nu$ & $\nu$ & 1 & 1 \\
\hline$G_{\mathrm{HW}}^{\prime}$ & $g_{13}$ & $g_{23}$ & $g_{23}$ & $\lambda$ & $\mu$ & 0 & $-\lambda-2 \mu$ & $\lambda$ & $-\lambda$ & $\mu+\nu$ & $\lambda+\mu+\nu$ & $\nu$ & 1 & 0 \\
\hline$G_{L W}$ & $g_{23}$ & $g_{33}$ & $g_{33}$ & 0 & 0 & 0 & $2 \lambda+\mu$ & $\mu$ & $-\mu$ & $\nu$ & $\mu+\nu$ & $\lambda+\mu+\nu$ & 0 & 0 \\
\hline$G_{L W}^{\prime}$ & $g_{23}$ & $g_{33}$ & $\begin{array}{l}g_{33} \\
g_{23}\end{array}$ & 0 & 0 & $\mu$ & $2 \lambda+\mu$ & $\mu$ & $\mu$ & $\mu+\nu$ & $\nu$ & $\lambda+\mu+\nu$ & 0 & 1 \\
\hline
\end{tabular}

the 3-direction is either a maximum or a minimum. The value of the subgroup labels for these so-called extremal states $\left(\left|G_{E}\right\rangle\right)$ are summarized by Table $\mathrm{I}$. The $I$ and $J$ labels form a convenient code by which the states can be distinguished. The labels $\lambda, \mu, \nu$ can therefore be thought of as either specifying or being specified by the distribution of oscillator quanta for extremal states.

In the $S U_{3} \supset R_{3}$ reduction states are labeled by the total angular momentum $L$ and its projection $M$. Multiple occurrences of a given $L$ can be distinguished in a variety of ways. 16 The physically most significant scheme is that due to Elliott in which case $K$, the projection of $L$ along the body-fixed 3 -axis, is used to sort the $L$-values into the familiar $K$-bands of rotational model theory. ${ }^{17}$ The prescription given is that projected states defined by

$$
|(G) K L M\rangle \equiv P_{H_{K}}|G\rangle \equiv(2 L+1) \int d \Omega D_{L_{K}}^{*}(\Omega) R(\Omega)|G\rangle
$$

form a complete basis if $G=G_{E}$ and for:

$$
\begin{aligned}
G_{E}=G_{H W}: & K=\lambda, \lambda-2, \ldots, 1 \text { or } 0, \\
& L=K, K+1, \ldots, K+\mu, \quad K \neq 0, \\
& L=\mu, \mu-2, \ldots, 1 \text { or } 0, \quad K=0 ; \\
G_{E}=G_{L W}: \quad & K=\mu, \mu-2, \ldots, 1 \text { or } 0, \\
& L=K, K+1, \ldots, K+\lambda, \quad K \neq 0, \\
& L=\lambda, \lambda-2, \ldots, 1 \text { or } 0, \quad K=0 .
\end{aligned}
$$

In Eq. (3), $D_{M K}^{L}(\Omega)$ is an $R_{3}$ rotation matrix and $R(\Omega)$ is an $R_{3}$ rotation operator. The integration is over Euler angles.

States defined by Eqs. (3)-(4) are not normalized nor are they orthogonal with respect to the $K$-label. Working within such a scheme leads ultimately to nonhermitian matrices. To avoid this complication, it is convenient to orthonormalize the basis using a Gram-Schmidt process. The physical interpretation of $K$ as a band label can be maintained approximately if a prescription analogous to that outlined by Vergados is used. ${ }^{18}$ In this case

$$
\left|\left(G_{E}\right) K_{i} L M\right\rangle=\sum_{j \leq i} o_{i j}\left|\left(G_{E}\right) K_{j} L M\right\rangle,
$$

where the orthonormalization matrix $O_{i j}$ is defined recursively by the formulas

$$
\begin{gathered}
O_{i i}=1 /\left(\left\langle\left(G_{E}\right) K_{i} L M \mid\left(G_{E}\right) K_{i} L M\right\rangle-\sum_{j<i} O_{j i} O_{j i}\right)^{1 / 2} \\
O_{j i}=O_{j j}\left(\left\langle\left(G_{E}\right) K_{j} L M \mid\left(G_{E}\right) K_{i} L M\right\rangle-\sum_{k<j<i} O_{k j} O_{k i}\right)^{1 / 2} \\
O_{i j}=O_{i i}\left(\delta_{i j}-\sum_{j \leq k<i} O_{k j} O_{k i}\right) .
\end{gathered}
$$

An analytic expression which allows the coefficients $\left\langle\left(G_{E}\right) K_{i} L M \mid\left(G_{E}\right) K_{j} L M\right\rangle$ to be evaluated is given in Sec. 3. Unlike the $\kappa$ of Vergados, $\nVdash$ like $K$ is given by either Eq. (4a) or Eq. (4b). The extent to which different $K$-values are mixed by the orthonormalization process depends upon the relative magnitude of the coefficients $\left\langle\left(G_{E}\right) K_{i} L M\right.$ $\left.\left(G_{E}\right) K_{i} L M\right\rangle$ and $\left\langle\left(G_{E}\right) K_{i} L M \mid\left(G_{E}\right) K_{j} L M\right\rangle$. It can be verified that the mixing is indeed small. In particular, for $G_{E}=$ $G_{\mathrm{HW}}\left(G_{\mathrm{LW}}\right)$ and $i \neq j\left\langle\left(G_{E}\right) K_{i} L M \mid\left(G_{E}\right) K_{j} L M\right\rangle \rightarrow 0$ if $\lambda(\mu)$ is fixed and $\mu(\lambda) \rightarrow \infty$.

\section{ALGEBRAIC FORMULATION}

If $\alpha$ represents a set of row labels used to distinguish orthonormal basis states within a given representation of $S U_{3}\left(\alpha=\epsilon \Lambda M_{\Lambda}\right.$, or $\mathcal{K} L M$, or $\left.\cdots\right)$, the Wigner coefficients $\left\langle\left(\lambda_{1} \mu_{1}\right) \alpha_{1} ;\left(\lambda_{2} \mu_{2}\right) \alpha_{2} \mid\left(\lambda_{3} \mu_{3}\right) \alpha_{3}\right\rangle_{0}$ are by definition the elements of a unitary transformation between coupled and uncoupled representations of $\mathrm{SU}_{3}$ in the $\alpha$-scheme,

$\left|\left(\lambda_{3} \mu_{3}\right) \alpha_{3}\right\rangle_{\rho}$
$=\sum_{\alpha_{1} \alpha_{2}}\left\langle\left(\lambda_{1} \mu_{1}\right) \alpha_{1} ;\left(\lambda_{2} \mu_{2}\right) \alpha_{2} \mid\left(\lambda_{3} \mu_{3}\right) \alpha_{3}\right\rangle_{\rho}\left|\left(\lambda_{1} \mu_{1}\right) \alpha_{1}\right\rangle\left|\left(\lambda_{2} \mu_{2}\right) \alpha_{2}\right\rangle$.

The outer multiplicity label $\rho=1,2, \ldots, \rho_{\max }$ is used to distinguish multiple occurrences of a given $\left(\lambda_{3} \mu_{3}\right)$ in the direct product $\left(\lambda_{1} \mu_{1}\right) \times\left(\lambda_{2} \mu_{2}\right)$. Although a definition bearing physical significance comparable, for example, to that associated with Elliott's choice of $K$ for a resolution of the inner multiplicity problem in the $S U_{3} \supset R_{3}$ reduction has not been proposed to fix $\rho$, Biedenharn and Louck and co-workers have demonstrated in a series of articles ${ }^{6-12}$ that a mathematically canonical definition which puts the outer multiplicity on a sound group theoretical basis can be obtained through the use of the labels of an upper Gel'fand pattern for a Wigner operator of irreducible tensor character $\left(\lambda_{2} \mu_{2}\right)$. The practical aspects of this choice are manifest in the vanishing of certain Wigner and Racah coefficients [Eqs. (15), (23), below], simple symmetry relations under conjugation [Eqs. (32)(36), below], and nice limiting properties for the $S U_{3} \supset$ $S U_{2} \times U_{1}$ Wigner coefficients (see Ref. 11, for example). Outlined below are techniques which exploit the essential features of this definition (albeit somewhat obscured but only so as to minimize notational needs) in defining an algorithm (based on an ordinary tensor formalism built with tensor operators which by construction have the same null space properties as the Wigner operators of Biedenharn and Louck) which can be used to evaluate all $S U_{3} \supset S U_{2} \times U_{1}$ Wigner coefficients. Note that for most practical purposes, however, the outer multiplicity can be considered fully labelled with a running index $\rho=1$, $2, \ldots, \rho_{\max }$ which distinguishes orthonormal basis states in the product space,

$$
\begin{aligned}
& \sum_{\alpha_{1} \alpha_{2}}\left\langle\left(\lambda_{1} \mu_{1}\right) \alpha_{1} ;\left(\lambda_{2} \mu_{2}\right) \alpha_{2} \mid\left(\lambda_{3} \mu_{3}\right) \alpha_{3}\right\rangle_{\rho} \\
& \times\left\langle\left(\lambda_{1} \mu_{1}\right) \alpha_{1} ;\left(\lambda_{2} \mu_{2}\right) \alpha_{2} \mid\left(\lambda_{3}^{\prime} \mu_{3}^{\prime}\right) \alpha_{3}\right\rangle_{\rho^{\prime}}=\delta_{\lambda_{3}^{\prime} \lambda_{3}{ }_{\mu_{3}^{\prime} \mu_{3}} \delta_{\rho^{\prime} \rho} .}
\end{aligned}
$$

\section{A. $S U_{3} \supset S U_{2} \times U_{1}$ Wigner coefficients}

Irreducible tensor operators under $S U_{3}, T^{(\lambda \mu)}$, can be defined through their commutation properties with the infinitesimal generators of the group. 19 The WignerEckart theorem allows one to express the matrix elements of tensor operators defined in this manner as a sum over $\rho$ of the product of a $\rho$-dependent generalized 
reduced matrix element multiplied by the corresponding Wigner coefficient. Specifically, for the $S U_{3} \supset S U_{2} \times U_{1}$ reduction,

$$
\begin{aligned}
& \left\langle\left(\lambda_{3} \mu_{3}\right) \epsilon_{3} \Lambda_{3} M_{\Lambda_{3}}\left|T_{\epsilon_{2} \kappa_{2} M_{\Lambda_{2}}}^{\left(\lambda_{2} \mu_{2}\right)}\right|\left(\lambda_{1} \mu_{1}\right) \epsilon_{1} \Lambda_{1} M_{\Lambda_{1}}\right\rangle \\
& =\sum_{\rho}\left\langle\left(\lambda_{3} \mu_{3}\right)\left\|T^{\left(\lambda_{2} \mu_{2}\right)}\right\|\left(\lambda_{1} \mu_{1}\right)\right\rangle_{\rho} \\
& \quad \times\left\langle\left(\lambda_{1} \mu_{1}\right) \epsilon_{1} \Lambda_{1} M_{\Lambda_{1}} ;\left(\lambda_{2} \mu_{2}\right) \epsilon_{2} \Lambda_{2} M_{\Lambda_{2}} \mid\left(\lambda_{3} \mu_{3}\right) \epsilon_{3} \Lambda_{3} M_{\Lambda_{3}}\right\rangle_{p}
\end{aligned}
$$

This result can be used to define Wigner coefficients through the matrix elements of specially chosen tensor operators $K^{\left(\lambda_{2} \mu_{2}\right)}(\rho)$,

$$
\begin{aligned}
& \left\langle\left(\lambda_{3} \mu_{3}\right) \epsilon_{3} \Lambda_{3} M_{\Lambda_{3}}\left|K_{\epsilon_{2}}^{\left(\lambda_{2} \mu_{2} \mu_{2} M_{\Lambda_{2}}\right.}(\rho)\right|\left(\lambda_{1} \mu_{1}\right) \epsilon_{1} \Lambda_{1} M_{\Lambda_{1}}\right\rangle \\
& =\left\langle\left(\lambda_{3} \mu_{3}\right)\left\|K^{\left(\lambda_{2} \mu_{2}{ }^{3}\right.}(\rho)\right\|\left(\lambda_{1} \mu_{1}\right)\right\rangle \\
& \quad \times\left\langle\left(\lambda_{1} \mu_{1}\right) \epsilon_{1} \Lambda_{1} M_{\Lambda_{1}} ;\left(\lambda_{2} \mu_{2}\right) \epsilon_{2} \Lambda_{2} M_{\Lambda_{2}} \mid\left(\lambda_{3} \mu_{3}\right) \epsilon_{3} \Lambda_{3} M_{\Lambda_{3}}\right\rangle_{\rho}
\end{aligned}
$$

for which the $\rho$-summation of Eq. (9) is not required. The generalized reduced matrix element $\left\langle\left(\lambda_{1} \mu_{1}\right) \| K^{\left(\lambda_{2}^{*} \mu_{2}\right)}\right.$ ( $\left.\rho) \|\left(\lambda_{3} \mu_{3}\right)\right\rangle$ is then just a normalization factor. In particular, the infinitesimal generators which have irreducible tensor character $\left(\lambda_{2} \mu_{2}\right)=(11)$ and operate only within a given representation of $S U_{3}$ [e.g., $\left(\lambda_{1} \mu_{1}\right)=$ $\left(\lambda_{3} \mu_{3}\right)=(\lambda \mu)$, only] are by definition matrix elements of the $\rho=1$ variety.

The problem is then one of constructing the operators $K^{\left(\lambda_{2} \mu_{2}\right)}(\rho)$; and in particular, constructing them in a manner which serves to uniquely define the outer multiplicity label $\rho$. The scheme is straightforward: Clearly $\rho_{\max }$, the number of occurrences of $\left(\lambda_{3} \mu_{3}\right)$ in the direct product $\left(\lambda_{1} \mu_{1}\right) \times\left(\lambda_{2} \mu_{2}\right)$, depends upon $\lambda_{1}, \mu_{1}, \lambda_{2}, \mu_{2}, \lambda_{3}, \mu_{3}$. It is also clear that there exists an $\eta$ such that $\left(\lambda_{3} \mu_{3}\right)$ occurs exactly $\rho$ times in the product $\left(\lambda_{1} \mu_{1}\right) \times\left(\lambda_{2}-\eta\right.$, $\left.\mu_{2}-\eta\right)$. And in this case $\rho$ depends upon $\lambda_{1}, \mu_{1}, \lambda_{2}-\eta$, $\mu_{2}-\eta, \lambda_{3}, \mu_{3}$. Let $\eta_{\max }$ be the value of $\eta$ such that $\left(\lambda_{1} \mu_{1}\right) \times\left(\lambda_{2}-\eta_{\max }, \mu_{2}-\eta_{\max }\right) \rightarrow\left(\lambda_{3} \mu_{3}\right)$ is not allowed whereas $\left(\lambda_{1} \mu_{1}\right) \times\left(\lambda_{2}-\eta_{\max }+1, \mu_{2}-\eta_{\max }+1\right) \rightarrow\left(\lambda_{3} \mu_{3}\right)$ occurs with a multiplicity of one. Then $\left(\lambda_{1} \mu_{1}\right) \times\left(\bar{\lambda}_{2} \equiv\right.$ $\left.\lambda_{2}-\eta_{\max }+\rho, \bar{\mu}_{2} \equiv \mu_{2}-\eta_{\max }+\rho\right) \rightarrow\left(\lambda_{3} \mu_{3}\right)$ occurs with a multiplicity of $\rho$ for $\rho=1,2, \ldots, \rho_{\max } \leq \eta_{\max }$. In this way, $\left(\lambda_{1} \mu_{1}\right) \times\left(\bar{\lambda}_{2} \vec{\mu}_{2}\right) \rightarrow\left(\lambda_{3} \mu_{3}\right)$ can be considered the parent coupling for the pth occurrence of $\left(\lambda_{3} \mu_{3}\right)$ in the product $\left(\lambda_{1} \mu_{1}\right) \times\left(\lambda_{2} \mu_{2}\right)$. The question then arises: Is it possible to construct the $K^{\left(\lambda_{2} \mu_{2}\right)}(\rho)$ from the corresponding $K^{\left(\bar{\lambda}_{2} \ddot{\mu}_{2}\right)}(\rho)$ in such a way as to preserve the unique null space) property of the parent operator which allow it to generate the $\rho$ th occurrence (and no more) of $\left(\lambda_{3} \mu_{3}\right)$ in the product space? The answer is yes, it can be done via a build-up process using the group generators $K^{(11)} \equiv$ $K^{(11)}(\rho=1)$. In particular, iterating the result

$$
\begin{aligned}
K_{\epsilon_{2} \Lambda_{2} M_{\Lambda_{2}}}^{\left(\lambda_{2} \mu_{2}\right)}(\tilde{\rho})= & {\left[K^{\left(\lambda_{2}-1, \mu_{2}-1\right)}(\rho) \times K^{(11)}\right]_{\epsilon_{2} \Lambda_{2} M_{\Lambda_{2}}}^{\left(\lambda_{2} \mu_{2}\right)} } \\
= & \sum_{\epsilon \Lambda \Lambda_{2}^{\prime} M_{\Lambda}}\left\langle(11) \epsilon \Lambda M_{\Lambda} ;\left(\lambda_{2}-1, \mu_{2}-1\right)\right. \\
& \epsilon_{2}^{\prime} \Lambda_{2}^{\prime} M_{\Lambda_{2}}^{\prime}\left|\left(\lambda_{2} \mu_{2}\right) \epsilon_{2} \Lambda_{2} M_{\Lambda_{2}}\right\rangle K_{\epsilon_{2}^{\prime} \Lambda_{2}^{\prime} M_{\Lambda_{2}}^{\prime}}^{\left(\lambda_{2}-1, \mu_{2}-1\right)}(\rho) K_{\epsilon \Lambda M_{\Lambda}}^{(11)}
\end{aligned}
$$

allows one to relate $K^{\left(\lambda_{2} \mu_{2}\right)}(\rho)$ to $K^{\left(\bar{\lambda}_{2} \bar{\mu}_{2}\right)}(\rho)$ for each $\rho$. Logical consistency demands, of course, that in each step $\tilde{\rho}$ be chosen numerically equal to $\rho$ and that $\rho=1$ corresponds to a multiplicity free parent coupling, $\rho=2$ to the second solution in the parent coupling having a twofold outer multiplicity, etc. The tilde, however, is used to denote the fact that $\rho$-orthogonality in the product space is not guaranteed; that is, $K^{\left(\lambda_{2} \mu_{2}\right)}(\bar{\rho})$ will in general be a linear combination of all $K^{\left(\lambda_{2} \mu_{2}\right.}(\rho)$ with $\rho \leq \tilde{\rho}$. (That operators with $\rho>\bar{\rho}$ are not generated is a consequence of the fact that the group generators preserve the null space properties of the parent operator. Further discussion on the consequence of this result is given below. In effect, it means that the weight diagram ${ }^{11}$ for a coupled operator of the type $T^{(\lambda \mu)} \times K^{(11)}$ is the same as for $T^{(\lambda \mu)}$.) To be sure, the build-up process cannot be used to define $K^{\left(\bar{\lambda}_{2} \bar{\mu}_{2}\right)}(\rho)$ because $K^{\left(\lambda_{2}-1, \bar{\mu}_{2}-1\right)}(\rho) \equiv 0$. But this presents no major problem since an analytic expression for the Wigner coefficients corresponding to the $p$ th occurrence of $\left(\lambda_{3} \mu_{3}\right)$ in the product $\left(\lambda_{1} \mu_{1}\right) \times\left(\bar{\lambda}_{2} \bar{\mu}_{2}\right)$ is available [Eq. (20), below] and through Eq. (10) serves to define the first nonvanishing operator in the build-up process. Note that the Wigner coefficient appearing in Eq. (11) is multiplicity free. Substitution of Eq. (11) into Eq. (10) yields

$$
\begin{aligned}
&\left\langle\left(\lambda_{1} \mu_{1}\right) \epsilon_{1} \Lambda_{1} M_{\Lambda_{1}} ;\left(\lambda_{2} \mu_{2}\right) \epsilon_{2} \Lambda_{2} M_{\Lambda_{2}} \mid\left(\lambda_{3} \mu_{3}\right) \epsilon_{3} \Lambda_{3} M_{\Lambda_{3}}\right\rangle_{\tilde{\rho}} \\
&=\left\langle\left(\lambda_{3} \mu_{3}\right)\left\|K^{\left(\lambda_{2} \mu_{2}\right)}(\tilde{\rho})\right\|\left(\lambda_{1} \mu_{1}\right)\right\rangle-1 \\
& \times\left\langle\left(\lambda_{3} \mu_{3}\right)\left\|K^{\left(\lambda_{2}-1, \mu_{2}-1\right)}(\rho)\right\|\left(\lambda_{1} \mu_{1}\right)\right\rangle\left\langle\left(\lambda_{1} \mu_{1}\right)\left\|K^{(1)}\right\|\left(\lambda_{1} \mu_{1}\right)\right\rangle \\
& \times \sum_{\epsilon \Lambda \Lambda_{1} \Lambda_{2}^{\prime} M_{\Lambda}}\left\langle(11) \epsilon \Lambda M_{\Lambda} ;\left(\lambda_{2}-1, \mu_{2}-1\right) \epsilon_{2}^{\prime} \Lambda_{2}^{\prime} M_{\Lambda_{2}}^{\prime}\right| \\
&\left.\left(\lambda_{2} \mu_{2}\right) \epsilon_{2} \Lambda_{2} M_{\Lambda_{2}}\right\rangle \\
& \times\left\langle\left(\lambda_{1} \mu_{1}\right) \epsilon_{1} \Lambda_{1} M_{\Lambda_{1}} ;(11) \epsilon \Lambda M_{\Lambda} \mid\left(\lambda_{1} \mu_{1}\right) \epsilon_{1}^{\prime} \Lambda_{1}^{\prime} M_{\Lambda_{1}^{\prime}}^{\prime}\right\rangle_{\rho}=1 \\
& \times\left\langle\left(\lambda_{1} \mu_{1}\right) \epsilon_{1}^{\prime} \Lambda_{1}^{\prime} M_{\Lambda_{1}^{\prime}}^{\prime} ;\left(\lambda_{2}-1, \mu_{2}-1\right) \epsilon_{2}^{\prime} \Lambda_{2}^{\prime} M_{\Lambda_{2}}^{\prime}\right| \\
&\left.\left(\lambda_{3} \mu_{3}\right) \epsilon_{3} \Lambda_{3} M_{\Lambda_{3}}\right\rangle_{\rho} .
\end{aligned}
$$

If $K^{(11)}$ were not chosen to be of the generator type, representations other than $\left(\lambda_{1} \mu_{1}\right)$ would appear on the right- and left-hand sides of the matrix elements of $K^{\left(\lambda_{2}-1, \mu_{2}-1\right)}(\rho)$ and $K^{(11)}$ and a summation over these representation labels would be required. Factoring each coupling coefficient into a reduced coefficient (doublebarred or isoscalar part) multiplied by an ordinary coefficient which carries the dependence upon the $S U_{2}$ projection labels and carrying out the summation over projection quantum numbers yields

$$
\begin{aligned}
&\left\langle\left(\lambda_{1} \mu_{1}\right) \epsilon_{1} \Lambda_{1} ;\left(\lambda_{2} \mu_{2}\right) \epsilon_{2} \Lambda_{2} \|\left(\lambda_{3} \mu_{3}\right) \epsilon_{3} \Lambda_{3}\right\rangle_{\tilde{\rho}} \\
&=\left\langle\left(\lambda_{3} \mu_{3}\right)\left\|K^{\left(\lambda_{2} \mu_{2}\right)}(\tilde{\rho})\right\|\left(\lambda_{1} \mu_{1}\right)\right\rangle^{-1} \\
& \times\left\langle\left(\lambda_{3} \mu_{3}\right)\left\|K^{\left(\lambda_{2}-1, \mu_{2}-1\right)}(\rho)\right\|\left(\lambda_{1} \mu_{1}\right)\right\rangle\left\langle\left(\lambda_{1} \mu_{1}\right)\left\|K^{(11)}\right\|\left(\lambda_{1} \mu_{1}\right)\right\rangle \\
& \times \sum_{\epsilon \Lambda \Lambda_{1}^{\prime} \Lambda_{2}^{\prime}}\left\langle(11) \epsilon \Lambda ;\left(\lambda_{2}-1, \mu_{2}-1\right) \epsilon_{2}^{\prime} \Lambda_{2}^{\prime} \|\left(\lambda_{2} \mu_{2}\right) \epsilon_{2} \Lambda_{2}\right\rangle \\
& \times\left\langle\left(\lambda_{1} \mu_{1}\right) \epsilon_{1} \Lambda_{1}^{\prime} ;(11) \epsilon \Lambda \|\left(\lambda_{1} \mu_{1}\right) \epsilon_{1}^{\prime} \Lambda_{1}^{\prime}\right\rangle_{\rho}=1 \\
& \times\left\langle\left(\lambda_{1} \mu_{1}\right) \epsilon_{1}^{\prime} \Lambda_{1}^{\prime} ;\left(\lambda_{2}-1, \mu_{2}-1\right) \epsilon_{2}^{\prime} \Lambda_{2}^{\prime} \|\left(\lambda_{3} \mu_{3}\right) \epsilon_{3} \Lambda_{3}\right\rangle_{\rho} \\
& \times U\left(\Lambda_{1} \Lambda \Lambda_{3} \Lambda_{2}^{\prime} ; \Lambda_{1}^{\prime} \Lambda_{2}\right)
\end{aligned}
$$

where $U\left(\Lambda_{1} \Lambda \Lambda_{3} \Lambda_{2}^{\prime} ; \Lambda_{1} \Lambda_{2}\right)$ is an ordinary $S U_{2}$ recoupling coefficient and $\epsilon_{1}=\epsilon_{3}-\epsilon_{2}, \epsilon_{2}^{\prime}=\epsilon_{2}-\epsilon, \epsilon_{1}^{\prime}=\epsilon_{3}-\epsilon_{2}$ $+\epsilon$.

It should be emphasized that Eq. (13) is valid for completely general arguments $\epsilon_{1}, \Lambda_{1}, \epsilon_{2}, \Lambda_{2}, \epsilon_{3}, \Lambda_{3}$ and, furthermore, that certain coupling coefficients derived using this expression must necessarily vanish identically. To see this, consider in more detail a coefficient calculated by repeating the recursion process $\eta$ times. The required matrix elements are for a tensor operator 


$$
\begin{aligned}
& K^{\left(\lambda_{2} \mu_{2}\right)}(\tilde{\rho}) \\
& \quad=\left[\cdots\left[\left[K^{\left(\lambda_{2}-\eta, \mu_{2}-\eta\right)}(\rho) \times K^{(11)}\right] \times K^{(11)}\right] \cdots \times K^{(11)}\right]^{\left(\lambda_{2} \mu_{2}\right)},
\end{aligned}
$$

in which $K^{(11)}$ appears $\eta$ times. In general the maximum change in $\Lambda$ induced by an operator $K^{(\lambda \mu)}$ is $\frac{1}{2}(\lambda+\mu)$ since this is the maximum value of $\Lambda$ in the representation $(\lambda \mu)$. The generators, however, are of a special type; they change $\Lambda$ by at most $\frac{1}{2}$. The operator given by Eq. (14) can therefore change $\Lambda$ by at most $\left(\lambda_{2}-\eta+\mu_{2}-\right.$ $\eta) / 2+\eta / 2=\left(\lambda_{2}+\mu_{2}-\eta\right) / 2$. Consequently, the corresponding coupling coefficient must be zero if $\left|. \Lambda_{1}-\Lambda_{3}\right|>$ $\frac{1}{2}\left(\lambda_{2}+\mu_{2}-\eta\right)$. The maximum $\eta$ for which this result is valid is simply $\eta=\eta_{\max }-\rho$. Consequently $\left\langle\left(\lambda_{1} \mu_{1}\right) \epsilon_{1} \Lambda_{1}\right.$; $\left.\left(\lambda_{2} \mu_{2}\right) \epsilon_{2} \Lambda_{2} \|\left(\lambda_{3} \mu_{3}\right) \epsilon_{3} \Lambda_{3}\right\rangle_{\tilde{p}}$ must vanish for $\left|\Lambda_{1}-\Lambda_{3}\right|>$ $\frac{1}{2}\left(\lambda_{2}+\mu_{2}-\eta_{\max }+\tilde{\rho}\right)$. This property is completely general and a direct consequence of the build-up process used to define the coefficients. Note that the number of coefficients predicted to be zero (more zeros may appear but for other reasons) is always a decreasing function of $\tilde{\rho}$. Although solutions obtained via repeated applications of Eq. (13) are not necessarily orthogonal with respect to the $\tilde{p}$-label, orthogonalizing in the increasing order $\rho=$ $1,2, \ldots, \rho_{\max }$ using a Gram-Schmidt process preserves the vanishings; and hence the Wigner coefficients satisfy

$$
\begin{aligned}
& \left\langle\left(\lambda_{1} \mu_{1}\right) \epsilon_{1} \Lambda_{1} ;\left(\lambda_{2} \mu_{2}\right) \epsilon_{2} \Lambda_{2} \|\left(\lambda_{3} \mu_{3}\right) \epsilon_{3} \Lambda_{3}\right\rangle_{\rho}=0 \\
& \quad \text { for }\left|\Lambda_{1}-\Lambda_{3}\right|>\frac{1}{2}\left(\lambda_{2}+\mu_{2}-\eta_{\max }+\rho\right) .
\end{aligned}
$$

This then quarantees the uniqueness of our result which by construction coincides with the Biedenharn and Louck prescription for a resolution of the outer multiplicity. Thus Eq. (13) provides a recursive means of defining the $S U_{3} \supset S U_{2} \times U_{1}$ Wigner coefficients for each mode of coupling characterized by $\rho$.

An expression which is computationally convenient to evaluate can be obtained from Eq. (13) by restricting $\epsilon_{2} \Lambda_{2}=$ HW and $\epsilon_{3} \Lambda_{3}=\mathrm{HW}$. In this case $\epsilon \Lambda$ and $\epsilon_{2}^{\prime} \Lambda_{2}^{\prime}$ are also forced to be of HW and $\left\langle(11) \mathrm{HW} ;\left(\lambda_{2}-1, \mu_{2}-1\right)\right.$ HW $\left.\|\left(\lambda_{2} \mu_{2}\right) \mathrm{HW}\right\rangle=1$. The sum in Eq. (13) then reduces to simply

$$
\begin{aligned}
\sum_{\Lambda_{1}^{\prime}}\left\langle\left(\lambda_{1} \mu_{1}\right) \epsilon_{1} \Lambda_{1} ;(11) \mathrm{HW} \|\left(\lambda_{1} \mu_{1}\right) \epsilon_{1}-3, \Lambda_{1}^{\prime}\right\rangle_{\rho}=1 \\
\quad \times\left\langle\left(\lambda_{1} \mu_{1}\right) \epsilon_{1}-3, \Lambda_{1}^{\prime} ;\left(\lambda_{2}-1, \mu_{2}-1\right) \mathrm{HW} \|\left(\lambda_{3} \mu_{3}\right) \mathrm{HW}\right\rangle_{\rho} \\
\quad \times U\left(\Lambda_{1}, \frac{1}{2}, \lambda_{3} / 2,\left(\lambda_{2}-1\right) / 2 ; \Lambda_{1}^{\prime}, \lambda_{2} / 2\right) .
\end{aligned}
$$

It follows (making use of results available, for example, in Refs. 19 and 20) that

$\left\langle\left(\lambda_{1} \mu_{1}\right) \epsilon_{1} \Lambda_{1} ;\left(\lambda_{2} \mu_{2}\right) \mathrm{HW} \|\left(\lambda_{3} \mu_{3}\right) \mathrm{HW}\right\rangle_{\bar{\rho}}$

$$
\begin{aligned}
& =N\left[-\left(\frac{R\left(p_{1}+1\right) A\left(\Lambda_{1}, \lambda_{2} / 2, \lambda_{3} / 2\right)\left[B\left(\Lambda_{1}, \lambda_{2} / 2, \lambda_{3} / 2\right)+1\right]}{\left(2 \Lambda_{1}+2\right)\left(2 \Lambda_{1}+1\right)}\right)^{1 / 2}\left\langle\left(\lambda_{1} \mu_{1}\right) \epsilon_{1}-3, \Lambda_{1}+\frac{1}{2} ;\left(\lambda_{2}-1, \mu_{2}-1\right) \mathrm{HW} \|\left(\lambda_{3} \mu_{3}\right) \mathrm{HW}\right\rangle_{\rho}\right. \\
& \left.+\left(\frac{S\left(q_{1}+1\right) C\left(\Lambda_{1}, \lambda_{2} / 2, \lambda_{3} / 2\right) D\left(\Lambda_{1}, \lambda_{2} / 2, \lambda_{3} / 2\right)}{\left(2 \Lambda_{1}+1\right)\left(2 \Lambda_{1}\right)}\right)^{1 / 2}\left\langle\left(\lambda_{1} \mu_{1}\right) \epsilon_{1}-3, \Lambda_{1}-\frac{1}{2} ;\left(\lambda_{2}-1, \mu_{2}-1\right) \mathrm{HW} \|\left(\lambda_{3} \mu_{3}\right) \mathrm{HW}\right\rangle_{\rho}\right], \\
& \begin{array}{ll}
A\left(\Lambda_{1}, \Lambda_{2}, \Lambda_{3}\right)=\Lambda_{2}+\Lambda_{3}-\Lambda_{1}, & N\left(\Lambda_{2}+\frac{1}{2}\right)=S\left(q_{2}\right), \\
B\left(\Lambda_{1}, \Lambda_{2}, \Lambda_{3}\right)=\Lambda_{3}+\Lambda_{1}-\Lambda_{2}, & N\left(\Lambda_{2}-\frac{1}{2}\right)=R\left(p_{2}\right) . \\
C\left(\Lambda_{1}, \Lambda_{2}, \Lambda_{3}\right)=\Lambda_{1}+\Lambda_{2}-\Lambda_{3}, & (18)
\end{array}
\end{aligned}
$$$$
D\left(\Lambda_{1}, \Lambda_{2}, \Lambda_{3}\right)=\Lambda_{1}+\Lambda_{2}+\Lambda_{3}+1 \text {, }
$$$$
R\left(p_{i}\right)=p_{i}\left(\lambda_{i}+1-p_{i}\right)\left(\mu_{i}+1+p_{i}\right) \text {, }
$$$$
S\left(q_{i}\right)=q_{i}\left(\mu_{i}+1-q_{i}\right)\left(\lambda_{i}+\mu_{i}+2-q_{i}\right),
$$

where $N$ is a normalization factor. This result allows the recursion process of Eq. (13) to be carried out within a very limited number of coefficients. The restriction $\epsilon_{3} \Lambda_{3}=\mathrm{HW}$, however, also demands that

$$
\begin{gathered}
\left\langle\left(\lambda_{1} \mu_{1}\right) \epsilon_{1} \Lambda_{1} ;\left(\lambda_{2} \mu_{2}\right) \epsilon_{2}+3, \Lambda_{2}^{\prime} \|\left(\lambda_{3} \mu_{3}\right) \mathrm{HW}\right\rangle \\
=\left(\frac{\left(2 \Lambda_{2}^{\prime}+1\right)}{\left(2 \Lambda_{1}+1\right)\left(2 \Lambda_{2}+1\right) N\left(\Lambda_{2}^{\prime}\right)}\right)^{1 / 2} \sum_{\Lambda_{1}^{\prime}=\Lambda_{1} \pm 1 / 2} \frac{X\left(\Lambda_{1}^{\prime}, \Lambda_{2}^{\prime}\right)}{\sqrt{2 \Lambda_{1}^{\prime}+1}} \\
\times\left\langle\left(\lambda_{1} \mu_{1}\right) \epsilon_{1}+3, \Lambda_{1}^{\prime} ;\left(\lambda_{2} \mu_{2}\right) \epsilon_{2} \Lambda_{2} \|\left(\lambda_{3} \mu_{3}\right) \mathrm{HW}\right\rangle, \\
X\left(\Lambda_{1}+\frac{1}{2}, \Lambda_{2}+\frac{1}{2}\right)=-\left\{S\left(q_{1}\right)[\right. \\
\left.\times\left(\Lambda_{1}, \Lambda_{2}, \lambda_{3} / 2\right)+\frac{1}{2}\right] \\
\left.\times\left[B\left(\Lambda_{1}, \Lambda_{2}, \lambda_{3} / 2\right)+\frac{1}{2}\right]\right\}^{1 / 2}, \\
X\left(\Lambda_{1}-\frac{1}{2}, \Lambda_{2}+\frac{1}{2}\right)=-\left\{R\left(p_{1}\right)\left[C\left(\Lambda_{1}, \Lambda_{2}, \lambda_{3} / 2\right)+\frac{1}{2}\right]\right. \\
\left.\times\left[D\left(\Lambda_{1}, \Lambda_{2}, \lambda_{3} / 2\right)+\frac{1}{2}\right]\right\}^{1 / 2}, \\
X\left(\Lambda_{1}-\frac{1}{2}, \Lambda_{2}-\frac{1}{2}\right)=+\left\{S\left(q_{1}\right)\left[C\left(\Lambda_{1}, \Lambda_{2}, \lambda_{3} / 2\right)+\frac{1}{2}\right]\right. \\
\left.\left.\times\left[D\left(\Lambda_{1}, \Lambda_{2}, \lambda_{3} / 2\right)+\frac{1}{2}\right]\right\}\right\}^{1 / 2}, \\
X\left(\Lambda_{1}-\frac{1}{2}, \Lambda_{2}-\frac{1}{2}\right)=-\left\{R\left(p_{1}\right)\left[A\left(\Lambda_{1}, \Lambda_{2}, \lambda_{3} / 2\right)+\frac{1}{2}\right]\right. \\
\left.\left.\times\left[B\left(\Lambda_{1}, \Lambda_{2}, \lambda_{3} / 2\right)+\frac{1}{2}\right]\right\}\right\}^{1 / 2},
\end{gathered}
$$

And knowing this additional result allows all coefficients of the type $\epsilon_{3} \Lambda_{3}=\mathrm{HW}$ to be determined. Coefficients with $\epsilon_{3} \Lambda_{3} \neq$ HW follow from the ordinary recursion formula

$$
\begin{aligned}
& \left\langle\left(\lambda_{1} \mu_{1}\right) \epsilon_{1} \Lambda_{1} ;\left(\lambda_{2} \mu_{2}\right) \epsilon_{2} \Lambda_{2} \|\left(\lambda_{3} \mu_{3}\right) \epsilon_{3} \Lambda_{3}\right\rangle \\
& =\frac{1}{N_{3}}\left(\sum_{\Lambda_{1}^{\prime}=\Lambda_{1} \pm 1 / 2} N_{1}\left(\frac{2 \Lambda_{3}^{\prime}+1}{2 \Lambda_{1}^{\prime}+1}\right)^{1 / 2} U\left(\Lambda_{2} \Lambda_{3}^{\prime} \Lambda_{1} \frac{1}{2} ; \Lambda_{1}^{\prime} \Lambda_{3}\right)\right. \\
& \quad \times\left\langle\left(\lambda_{1} \mu_{1}\right) \epsilon_{1}-3, \Lambda_{1}^{\prime} ;\left(\lambda_{2} \mu_{2}\right) \epsilon_{2} \Lambda_{2} \|\left(\lambda_{3} \mu_{3}\right) \epsilon_{3}-3, \Lambda_{3}^{\prime}\right\rangle \\
& \quad+\sum_{\Lambda_{2}^{\prime}=\Lambda_{2} \pm 1 / 2} N_{2}\left(\frac{2 \Lambda_{3}+1}{2 \Lambda_{2}+1}\right)^{1 / 2} U\left(\Lambda_{1} \Lambda_{2}^{\prime} \Lambda_{3} \frac{1}{2} ; \Lambda_{3}^{\prime} \Lambda_{2}\right) \\
& \left.\quad \times\left\langle\left(\lambda_{1} \mu_{1}\right) \epsilon_{1} \Lambda_{1} ;\left(\lambda_{2} \mu_{2}\right) \epsilon_{2}-3, \Lambda_{2}^{\prime} \|\left(\lambda_{3} \mu_{3}\right) \epsilon_{3}-3, \Lambda_{3}^{\prime}\right\rangle\right), \\
& N_{i}= \begin{cases}\sqrt{S\left(q_{i}+1\right)}, & \Lambda_{i}-\Lambda_{i}^{\prime}=\frac{1}{2}, \\
\sqrt{R\left(q_{i}+1\right)}, & \Lambda_{i}-\Lambda_{i}^{\prime}=-\frac{1}{2} .\end{cases}
\end{aligned}
$$

The process is easily realized for small values of $n \equiv\left[\left(\lambda_{1}+\lambda_{2}-\lambda_{3}\right)-2\left(\mu_{1}+\mu_{2}-\mu_{3}\right)\right] / 3$. The maximum possible multiplicity is $n+1$, i.e., $\rho_{\max } \leq n+1$. For example, for an allowed coupling with $n=0, \rho_{\max }$ must be one and $\left\langle\left(\lambda_{1} \mu_{1}\right) \mathrm{HW} ;\left(\lambda_{2} \mu_{2}\right) \mathrm{HW} \|\left(\lambda_{3} \mu_{3}\right) \mathrm{HW}\right\rangle=1$. For $n=1, \rho_{\max }$ may be either one or two. If $\rho_{\max }=2$, the coefficients with $\rho=1$ and $\epsilon_{3} \Lambda_{3}=$ HW are determined via Eqs. (17) $-(18)$ from the result for $\left\langle\left(\lambda_{1} \mu_{1}\right) \mathrm{HW} ;\left(\lambda_{2}-\right.\right.$ $\left.\left.1, \mu_{2}-1\right) \mathrm{HW} \|\left(\lambda_{3} \mu_{3}\right) \mathrm{HW}\right\rangle$. The solution for $\rho=2$ can then be determined from Eq. (20) below. If, on the other hand, $\rho_{\max }=1$, either $\left(\lambda_{1} \mu_{1}\right) \times\left(\lambda_{2}-1, \mu_{2}-1\right) \rightarrow\left(\lambda_{3} \mu_{3}\right)$ 
is allowed and Eq. (20) cannot be used to generate an additional independent solution or $\left(\lambda_{1} \mu_{1}\right) \times\left(\lambda_{2}-1, \mu_{2}-\right.$ 1) $\rightarrow\left(\lambda_{3} \mu_{3}\right)$ is not allowed and Eq. (20) provides the only solution. For $n=2, \rho_{\max }$ may be either one, two, or three. And in this case it is still possible to generate useful algebraic results. For $n>2$, however, the recursion process yields unwieldy expressions making the algebraic approach extremely difficult if not impossible.
However, from the systematics of the results it is possible to predict a general algebraic expression for $\left\langle\left(\lambda_{1} \mu_{1}\right) \mathrm{HW} ;\left(\bar{\lambda}_{2} \bar{\mu}_{2}\right) \epsilon_{2} \Lambda_{2} \|\left(\lambda_{3} \mu_{3}\right) \mathrm{HW}\right\rangle_{\rho}\left(\bar{\lambda}_{2}=\lambda_{2}-\eta_{\max }+\rho\right.$, $\bar{\mu}_{2}=\mu_{2}-\eta_{\max }+\rho$ implying that $\rho$ is the maximum multiplicity for this coupling) which leads to coefficients that are automatically orthogonal to those obtained via Eq. (13) for the same $\bar{\lambda}_{2}, \bar{\mu}_{2}$ but lesser $\rho$. Explicitly, if $\tilde{p}=\mu-q$ and $\tilde{q}=\lambda-p$,

$$
\begin{aligned}
& \left\langle\left(\lambda_{1} \mu_{1}\right) \mathrm{HW} ;\left(\bar{\lambda}_{2} \bar{\mu}_{2}\right) \epsilon_{2} \Lambda_{2} \|\left(\lambda_{3} \mu_{3}\right) H W\right\rangle_{\rho}=N F\left(\tilde{\bar{p}}_{2}\right)\left[G\left(\tilde{\bar{q}}_{2}\right) / H\left(\tilde{\bar{q}}_{2}\right)\right]^{1 / 2} \text {, } \\
& F\left(\tilde{\bar{p}}_{2}\right)=\left\{\begin{array}{cc}
1, & \tilde{\bar{p}}_{2}=0, \\
(-1)^{\tilde{\bar{p}}_{2}} \sum_{i=0}\left(\begin{array}{c}
\tilde{\bar{p}}_{2} \\
i
\end{array}\right) \prod_{j=0}^{\tilde{\bar{p}}_{2}-1} f(j), & \tilde{\bar{p}}_{2} \geq 1,
\end{array}\right. \\
& f(j)= \begin{cases}\left(\bar{p}_{2}+j+1\right)\left(\mu_{1}+\bar{\lambda}_{2}+\bar{\mu}_{2}-n+j+2\right), & j<i, \\
(a+j+1)(b-j-1), & j \geq i,\end{cases} \\
& G\left(\tilde{\bar{q}}_{2}\right)= \begin{cases}\left(\bar{\lambda}_{2}-2 \tilde{\bar{q}}_{2}+n+1\right)\left(\begin{array}{c}
n \\
\tilde{\bar{q}}_{2}
\end{array}\right)_{j=j_{1}}^{j_{2}-1} g(j), & j_{2}>j_{1}, \\
1, & j_{2}=j_{1},\end{cases} \\
& g(j)= \begin{cases}(a+n-j)(b-n+j)(c+n-j)(d+n-j)\left(\lambda_{2}+\bar{\mu}_{2}-j+1\right), & j<\overline{\bar{q}}_{2}, \\
\bar{\mu}_{2}-n+j+1, & j \geq \overline{\bar{q}}_{2},\end{cases} \\
& \left.\begin{array}{l}
j_{1}=\text { minimum } \tilde{\bar{q}}_{2} \\
j_{2}=\text { maximum } \tilde{\tilde{q}}_{2}
\end{array}\right\} \text { for which the coupling } \Lambda_{1}+\Lambda_{2}=\Lambda_{3} \text { is allowed, } \\
& H\left(\tilde{\bar{q}}_{2}\right)=\left(\begin{array}{c}
n+1+\bar{\lambda}_{2}-\tilde{\bar{q}}_{2} \\
\bar{\lambda}_{2}-\tilde{\bar{q}}_{2}
\end{array}\right), \\
& a=A\left(\lambda_{1} / 2, \lambda_{2} / 2, \lambda_{3} / 2\right)-n / 2, \quad b=B\left(\lambda_{1} / 2, \lambda_{2} / 2, \lambda_{3} / 2\right)+n / 2+1, \\
& c=C\left(\lambda_{1} / 2, \lambda_{2} / 2, \lambda_{3} / 2\right)-n / 2, \quad d=D\left(\lambda_{1} / 2, \lambda_{2} / 2, \lambda_{3} / 2\right)-n / 2 \text {, } \\
& n=\left[\left(\lambda_{1}+\bar{\lambda}_{2}-\lambda_{3}\right)+2\left(\mu_{1}+\bar{\mu}_{2}-\mu_{3}\right)\right] / 3 \text {, }
\end{aligned}
$$

where $N$ is again the normalization factor. The formula [which is essentially the inverse of Eq. (18)]

$$
\begin{aligned}
& \left\langle\left(\lambda_{1} \mu_{1}\right) \epsilon_{1}+3, \Lambda_{1}^{\prime} ;\left(\lambda_{2} \mu_{2}\right) \epsilon_{2} \Lambda_{2} \|\left(\lambda_{3} \mu_{3}\right) \mathrm{HW}\right\rangle \\
& =\left(\frac{\left(2 \Lambda_{1}^{\prime}+1\right)}{\left(2 \Lambda_{1}+1\right)\left(2 \Lambda_{2}+1\right) N\left(\Lambda_{1}^{\prime}\right)}\right)^{1 / 2} \sum_{\Lambda_{2}^{\prime}=\Lambda_{2} \pm 1 / 2} \frac{Y\left(\Lambda_{1}^{\prime}, \Lambda_{2}^{\prime}\right)}{\sqrt{2 \Lambda_{2}^{\prime}+1}} \\
& \times\left\langle\left(\lambda_{1} \mu_{1}\right) \epsilon_{1} \Lambda_{1} ;\left(\epsilon_{2} \Lambda_{2}\right) \epsilon_{2}+3, \Lambda_{2}^{\prime} \|\left(\lambda_{3} \mu_{3}\right) \mathrm{HW}\right\rangle \text {, } \\
& Y\left(\Lambda_{1}+\frac{1}{2}, \Lambda_{2}+\frac{1}{2}\right)=-\left\{S\left(q_{2}\right)\left[A\left(\Lambda_{1}, \Lambda_{2}, \lambda_{3} / 2\right)+\frac{1}{2}\right]\right. \\
& \left.\times\left[B\left(\Lambda_{1}, \Lambda_{2}, \lambda_{3} / 2\right)+\frac{1}{2}\right]\right\}^{1 / 2} \text {, } \\
& Y\left(\Lambda_{1}+\frac{1}{2}, \Lambda_{2}-\frac{1}{2}\right)=+\left\{R\left(p_{2}\right)\left[C\left(\Lambda_{1}, \Lambda_{2}, \lambda_{3} / 2\right)+\frac{1}{2}\right]\right. \\
& \left.\times\left[D\left(\Lambda_{1}, \Lambda_{2}, \lambda_{3} / 2\right)+\frac{1}{2}\right]\right\}^{1 / 2}, \\
& Y\left(\Lambda_{1}-\frac{1}{2}, \Lambda_{2}+\frac{1}{2}\right)=-\left\{S\left(q_{2}\right)\left[C\left(\Lambda_{1}, \Lambda_{2}, \lambda_{3} / 2\right)+\frac{1}{2}\right]\right. \\
& \left.\times\left[D\left(\Lambda_{1}, \Lambda_{2}, \lambda_{3} / 2\right)+\frac{1}{2}\right]\right\}^{1 / 2}, \\
& Y\left(\Lambda_{1}-\frac{1}{2}, \Lambda_{2}-\frac{1}{2}\right)=-\left\{R\left(p_{2}\right)\left[A\left(\Lambda_{1}, \Lambda_{2}, \lambda_{3} / 2\right)+\frac{1}{2}\right]\right. \\
& \left.\times\left[B\left(\Lambda_{1}, \Lambda_{2}, \lambda_{3} / 2\right)+\frac{1}{2}\right]\right\}^{1 / 2}, \\
& N\left(\Lambda_{1}+\frac{1}{2}\right)=S\left(q_{1}\right), \\
& N\left(\Lambda_{1}-\frac{1}{2}\right)=R\left(p_{1}\right),
\end{aligned}
$$

can be used to generate coefficients with $\epsilon_{1} \Lambda_{1} \neq$ HW recursively. Note that Eq. (20) is valid for all $\rho$; it can be used to provide the starting coefficients for the recursion process.

The computational algorithm is then clear: Neglecting normalization factors, for each $\rho=1,2, \ldots, \rho_{\max }$, (i) start with the $\left.\left\langle\left(\lambda_{1} \mu_{1}\right) \mathrm{HW} ; \bar{\lambda}_{2} \bar{\mu}_{2}\right) \epsilon_{2} \Lambda_{2} \|\left(\lambda_{3} \mu_{3}\right) \mathrm{HW}\right\rangle_{\rho}$ of Eq. (20) and use Eq. (21) to generate the $\left\langle\left(\lambda_{1} \mu_{1}\right) \epsilon_{1} \Lambda_{1}\right.$; $\left.\left(\bar{\lambda}_{2} \bar{\mu}_{2}\right) \mathrm{HW} \|\left(\lambda_{3} \mu_{3}\right) \mathrm{HW}\right\rangle_{\rho}$, (ii) make use of Eq. (17) to generate the $\left\langle\left(\lambda_{1} \mu_{1}\right) \epsilon_{1} \Lambda_{1} ;\left(\lambda_{2} \mu_{2}\right) \mathrm{HW} \|\left(\lambda_{3} \mu_{3}\right) \mathrm{HW}\right\rangle_{\overline{0}}$ from the $\left\langle\left(\lambda_{1} \mu_{1}\right) \epsilon_{1} \Lambda_{1} ;\left(\lambda_{2} \bar{\mu}_{2}\right) \mathrm{HW} \|\left(\lambda_{3} \mu_{3}\right) \mathrm{HW}\right\rangle_{\rho}$, and (iii) obtain the $\left\langle\left(\lambda_{1} \mu_{1}\right) \epsilon_{1} \Lambda_{1} ;\left(\lambda_{2} \mu_{2}\right) \epsilon_{2} \Lambda_{2} \|\left(\lambda_{3} \mu_{3}\right) H W\right\rangle_{\bar{\rho}}$ by using Eq. (18) to step the $\epsilon_{2} \Lambda_{2}$ labels. Then (iv) use Eq. (8) with $\alpha_{3}=$ $\epsilon_{3} \Lambda_{3}=H W$ to orthonormalize the resultant coefficients in the increasing order $\rho=1,2, \ldots, \rho_{\max }$ and, depending upon need, and (v) obtain the $\left\langle\left(\lambda_{1} \mu_{1}\right) \epsilon_{1} \Lambda_{1} ;\left(\lambda_{2} \mu_{2}\right) \epsilon_{2} \Lambda_{2} \|\right.$ $\left.\left(\lambda_{3} \mu_{3}\right) \epsilon_{3} \Lambda_{3}\right\rangle_{\rho}$ by using Eq. (19) to step the $\epsilon_{3} \Lambda_{3}$ labels.

The process serves to define $S U_{3} \supset S U_{2} \times U_{1}$ Wigner coefficients to within an overall phase. The simplest and most natural way for fixing the phase is to take all the normalization factors involved in the process to be positive, and we adopt this convention. This is very different from the ordinary procedure in which a particular coefficient is assigned to be positive for each mode of coupling, i.e., each $\rho$-label. 21 With the current approach, however, it is difficult to predict the sign of each individual coefficient, making a priori introduction of the ordinary convention practically impossible. Of course, the technique outlined above allows the ordinary convention to be introduced $a$ posteriori during the orthonormalization process. And such a choice reflects it- 
self in the $\rho$-dependence of the symmetry properties of the Wigner coefficients (see Sec.4).

It is interesting to note the effect of changing the order of the coupling in Eq. (11),

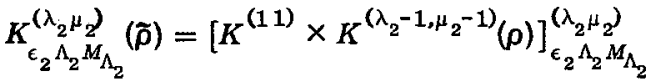

$$
\begin{aligned}
& =\sum_{\epsilon \Lambda \Lambda_{2}^{\prime} M_{\Lambda}}\left\langle\left(\lambda_{2}-1, \mu_{2}-1\right) \epsilon_{2}^{\prime} \Lambda_{2}^{\prime} M_{\Lambda_{2}}^{\prime} ;(11) \epsilon \Lambda M_{\Lambda} \mid\left(\lambda_{2} \mu_{2}\right) \epsilon_{2} \Lambda_{2} M_{\Lambda_{2}}\right\rangle
\end{aligned}
$$

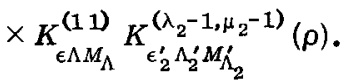

For this form, the result corresponding to Eq. (13) is

$$
\begin{aligned}
\left\langle\left(\lambda_{1} \mu_{1}\right) \epsilon_{1} \Lambda_{1} ;\left(\lambda_{2} \mu_{2}\right) \epsilon_{2} \Lambda_{2} \|\left(\lambda_{3} \mu_{3}\right) \epsilon_{3} \Lambda_{3}\right\rangle_{\tilde{\rho}} \\
=\left\langle\left(\lambda_{3} \mu_{3}\right)\left\|K^{\left(\lambda_{2} \mu_{2}\right)}(\tilde{\rho})\right\|\left(\lambda_{1} \mu_{1}\right)\right\rangle^{-1}\left\langle\left(\lambda_{3} \mu_{3}\right)\left\|K^{(11)}\right\|\left(\lambda_{3} \mu_{3}\right)\right\rangle \\
\quad \times\left\langle\left(\lambda_{3} \mu_{3}\right)\left\|K^{\left(\lambda_{2}-1, \mu_{2}-1\right)}(\rho)\right\|\left(\lambda_{1} \mu_{1}\right)\right\rangle \\
\quad \times \sum_{\epsilon \Lambda \Lambda_{2}^{\prime} \Lambda_{3}^{\prime}}\left\langle\left(\lambda_{2}-1, \mu_{2}-1\right) \epsilon_{2}^{\prime} \Lambda_{2}^{\prime} ;(11) \epsilon \Lambda \|\left(\lambda_{2} \mu_{2}\right) \epsilon_{2} \Lambda_{2}\right\rangle \\
\quad \times\left\langle\left(\lambda_{3} \mu_{3}\right) \epsilon_{3}^{\prime} \Lambda_{3}^{\prime} ;(11) \epsilon \Lambda \|\left(\lambda_{3} \mu_{3}\right) \epsilon_{3} \Lambda_{3}\right\rangle_{\rho=1} \\
\quad \times\left\langle\left(\lambda_{1} \mu_{1}\right) \epsilon_{1} \Lambda_{1} ;\left(\lambda_{2}-1, \mu_{2}-1\right) \epsilon_{2}^{\prime} \Lambda_{2}^{\prime} \|\left(\lambda_{3} \mu_{3}\right) \epsilon_{3}^{\prime} \Lambda_{3}^{\prime}\right\rangle_{\rho} \\
\quad \times U\left(\Lambda_{1} \Lambda_{2}^{\prime} \Lambda_{3} \Lambda_{3} \Lambda_{3}^{\prime} \Lambda_{2}\right) .
\end{aligned}
$$

The choice $\epsilon_{1} \Lambda_{1}=$ HW and $\epsilon_{2} \Lambda_{2}=\mathrm{LW}$ rather than $\epsilon_{2} \Lambda_{2}$ $=\mathrm{HW}$ and $\epsilon_{3} \Lambda_{3}=\mathrm{HW}$ can then be used to obtain a recursion relationship analogous to Eq. (17).

\section{B. $\mathrm{SU}_{3}$ Racah coefficients}

A straightforward generalization of the relationships between $\mathrm{SU}_{2}$ unitary recoupling coefficients and $\mathrm{SU}_{2}$ Wigner coefficients leads to the corresponding relationships between $S U_{3}$ unitary recoupling (Racah or $U$ functions) and $\mathrm{SU}_{3}$ Wigner coefficients. ${ }^{19}$ The most practical of these relationships for evaluating recoupling coefficients in terms of known Wigner coefficients is

$$
\begin{aligned}
& \sum\left\langle\left(\lambda_{1} \mu_{1}\right) \epsilon_{1} \Lambda_{1} ;\left(\lambda_{23} \mu_{23}\right) \epsilon_{23} \Lambda_{23} \|(\lambda \mu) \epsilon \Lambda\right\rangle_{\rho_{1,23}} \\
& \quad \times U\left(\lambda_{1} \mu_{1}\right)\left(\lambda_{2} \mu_{2}\right)(\lambda \mu)\left(\lambda_{3} \mu_{3}\right) ;\left(\lambda_{12} \mu_{12}\right) \rho_{12}, \\
&\left.\times \rho_{12,3}\left(\lambda_{23} \mu_{23}\right) \rho_{23}, \rho_{1,23}\right) \\
&= \sum_{\epsilon_{2} \Lambda_{2} \Lambda_{3} \Lambda_{12}}\left\langle\left(\lambda_{1} \mu_{1}\right) \epsilon_{1} \Lambda_{1} ;\left(\lambda_{2} \mu_{2}\right) \epsilon_{2} \Lambda_{2} \|\left(\lambda_{12} \mu_{12}\right) \epsilon_{12} \Lambda_{12}\right\rangle_{\rho_{12}} \\
& \times\left\langle\left(\lambda_{12} \mu_{12}\right) \epsilon_{12} \Lambda_{12} ;\left(\lambda_{3} \mu_{3}\right) \epsilon_{3} \Lambda_{3} \|(\lambda \mu) \epsilon \Lambda\right\rangle_{\rho_{12,3}} \\
& \times\left\langle\left(\lambda_{2} \mu_{2}\right) \epsilon_{2} \Lambda_{2} ;\left(\lambda_{3} \mu_{3}\right) \epsilon_{3} \Lambda_{3} \|\left(\lambda_{23} \mu_{23}\right) \epsilon_{23} \Lambda_{23}\right\rangle_{\rho_{23}} \\
& \times U\left(\Lambda_{1} \Lambda_{2} \Lambda_{3} ; \Lambda_{12} \Lambda_{23}\right) .
\end{aligned}
$$

Fixing $\epsilon_{1} \Lambda_{1}=H W$ and $\epsilon \Lambda=H W$ in this expression while letting $\Lambda_{23}$ run over its range of allowed values yields a set of simultaneous equations the solution of which is the required $U$ functions. Note that the choice $\epsilon_{1} \Lambda_{1}=\mathrm{HW}$ and $\epsilon \Lambda=H W$ makes it possible to evaluate all but one of the Wigner coefficients in Eq. (22) through Eqs. (17)-(18); the other requires Eq. (19) in addition.

The sum on the right-hand side of Eq. (13) can with the help of Eq. (22) be identified (apart from orthogonality) as simply

$$
\begin{gathered}
\sum_{\rho_{D}}\left\langle\left(\lambda_{1} \mu_{1}\right) \epsilon_{1} \Lambda_{1} ;\left(\lambda_{2} \mu_{2}\right) \epsilon_{2} \Lambda_{2} \|\left(\lambda_{3} \mu_{3}\right) \epsilon_{3} \Lambda_{3}\right\rangle_{\rho_{0}} U\left(\left(\lambda_{1} \mu_{1}\right)(11)\left(\lambda_{3} \mu_{3}\right)\right. \\
\left.\quad \times\left(\lambda_{2}-1, \mu_{2}-1\right) ;\left(\lambda_{1} \mu_{1}\right) \rho_{A}=1, \rho_{B}\left(\lambda_{2} \mu_{2}\right) \rho_{C}=1, \rho_{D}\right) .
\end{gathered}
$$

This is a direct consequence of the special character of the couplings involved in the product tensors of Eq. (11). More general couplings would, by analogy with $S U_{2}$, require a $9-(\lambda \mu)$ symbol. 22 The recursion formula (13) could therefore, in retrospect, be obtained from Eq. (22) by requiring $U\left(\left(\lambda_{1} \mu_{1}\right)(11)\left(\lambda_{3} \mu_{3}\right)\left(\lambda_{2}-1, \mu_{2}-1\right) ;\left(\lambda_{1} \mu_{1}\right) \rho_{A}=\right.$ $\left.1, \rho_{B}\left(\lambda_{2} \mu_{2}\right) \rho_{C}=1, \rho_{D}\right)=0$ for $\rho_{D} \neq \rho_{B}$. And indeed, this suggests a simple method by which the techniques developed in this article may be generalized to other group structures. Note that the orthonormalization process, if carried out in the increasing order $\rho=1,2, \ldots$, $\rho_{\max }$, maintains the zero value of the $U$ function for $\rho_{D}>$ $\rho_{B}$. Consequently,

$$
\begin{array}{r}
U\left(\left(\lambda_{1} \mu_{1}\right)(11)\left(\lambda_{3} \mu_{3}\right)\left(\lambda_{2}-1, \mu_{2}-1\right) ;\left(\lambda_{1} \mu_{1}\right) \rho_{A}=1\right. \\
\left.\rho_{B}\left(\lambda_{2} \mu_{2}\right) \rho_{C}=1, \rho_{D}\right)=0 \text { for } \rho_{D}>\rho_{B} .
\end{array}
$$

This result also follows from property (15) and is a direct consequence of the Biedenharn and Louck prescription for specifying the outer multiplicity.

\section{C. $S U_{3} \supset R_{3}$ Wigner coefficients}

The coefficients which effect the transformation between the $\epsilon \Lambda M_{\Lambda}$ and $K L M$ schemes are known. ${ }^{23}$ [The choice made in Eq. (3) requires that an additional factor of $2 L+1$ be included in evaluating Eq. (35) of Ref. 23 . In addition, including the phase factor $(i)^{n_{1}+n_{3}}$ in the definition of $|G\rangle$ makes the coefficient real.] Explicitly, if

$$
\left|\left(G_{E}\right) \mathcal{K} L M\right\rangle=\sum_{g}\left\langle G \mid\left(G_{E}\right) \mathcal{K} L M\right\rangle|G\rangle,
$$

$$
\left\langle G \mid\left(G_{E}\right) \mathcal{K}_{i} L M\right\rangle=\sum_{j \leq i} O_{i j}\left\langle G \mid\left(G_{E}\right) K_{j} L M\right\rangle,
$$

where $O_{i j}$ is the orthonormalization matrix of Eq. (6) and $\left\langle G \mid\left\langle G_{E}\right) K L M\right\rangle$ is the inner product of a state $|G\rangle$ [defined by Eq. (2)] with a state $\left|\left(G_{E}\right) K L M\right\rangle$ [defined by Eqs. (3)(4)]. The parameter $g$ in Eq. (24) is used to denote the subgroup labels $\left(g_{12}, g_{22}, g_{11} \sim p, q, r\right)$ of $G$. In terms of summation ( $K \rightarrow M, M \rightarrow M^{\prime}$ for reasons of symmetry),

$$
\begin{aligned}
& \left\langle G \mid\left(G_{\mathrm{HW}}\right) M L M^{\prime}\right\rangle=C \sum_{\gamma=0}^{p}\left(\begin{array}{l}
p \\
\gamma
\end{array}\right) S_{1}\left(M_{\Lambda}^{\prime} \Lambda^{\prime} N_{\Lambda}^{\prime} M^{\prime}\right) S_{1}\left(N_{\Lambda} \Lambda M_{\Lambda}=\Lambda M\right) S_{2}\left(\kappa^{\prime} k \kappa=k M^{\prime} L M\right), \\
& C=(-1)^{L-p}\left(\frac{2 L+1}{2^{p}}\right)^{2}\left[\left(\begin{array}{l}
\lambda \\
p
\end{array}\right)\left(\begin{array}{c}
\mu \\
q
\end{array}\right)\left(\begin{array}{c}
\lambda+\mu+1 \\
q
\end{array}\right)\left(\begin{array}{c}
2 L \\
L-M
\end{array}\right) /\left(\begin{array}{c}
2 L \\
L-M^{\prime}
\end{array}\right)\left(\begin{array}{c}
2 \Lambda^{\prime} \\
\Lambda^{\prime}+M_{\Lambda}^{\prime}
\end{array}\right)\left(\begin{array}{c}
p+\mu+1 \\
q
\end{array}\right)\right]^{1 / 2}, \\
& S_{1}\left(M_{\Lambda} \Lambda N_{\Lambda} M\right)=\sum_{\alpha}\left(\begin{array}{c}
\Lambda-N_{\Lambda} \\
\alpha
\end{array}\right)\left(\begin{array}{c}
\Lambda+N_{\Lambda} \\
\Lambda-M_{\Lambda}-\alpha
\end{array}\right) \sum_{\beta}(-1)^{\beta}\left(\begin{array}{c}
2 \Lambda-M_{\Lambda}-N_{\Lambda}-2 \alpha \\
\beta
\end{array}\right)\left(\begin{array}{c}
2 \alpha+M_{\Lambda}+N_{\Lambda} \\
\Lambda+M / 2-\beta
\end{array}\right), \\
& S_{2}\left(\kappa^{\prime} k \kappa M^{\prime} L M\right) \\
& =\frac{1}{2 k+L+1} \sum_{\alpha}(-1)^{\alpha}\left(\begin{array}{c}
L-M \\
\alpha
\end{array}\right)\left(\begin{array}{c}
L+M \\
L-M^{\prime}-\alpha
\end{array}\right) \sum_{\beta}(-1)^{\beta}\left(\begin{array}{c}
k+\kappa^{\prime} \\
\beta
\end{array}\right) /\left(\begin{array}{c}
2 k+L \\
q+\Lambda+M / 2+\Lambda^{\prime}+M^{\prime} / 2+\alpha-\beta-\gamma
\end{array}\right) \\
& \Lambda^{\prime}=(p+\mu-q) / 2, \quad \Lambda=\lambda / 2, \quad k=(\lambda+\mu-\gamma) / 2 \text {, } \\
& M_{\Lambda}^{\prime}=r-\Lambda^{\prime}, \quad M_{\Lambda}=\Lambda, \quad \kappa=k \text {, } \\
& N_{\Lambda}^{\prime}=p-\gamma-\Lambda^{\prime}, \quad N_{\Lambda}=\Lambda-\gamma, \quad k^{\prime}=k-(\lambda-p+\mu-q) \text {. }
\end{aligned}
$$


The corresponding expression for $\left\langle G \mid\left(G_{L W}\right) K L M\right\rangle$ can be obtained by conjugation (see Sec. 4). Note in particular that the overlap of two projected states required for a determination of the orthonormalization matrix $O_{i j}$, is given by

$$
\left\langle\left(G_{E}\right) K^{\prime} L M \mid\left(G_{E}\right) K L M\right\rangle=\left\langle G_{E} \mid\left(G_{E}\right) K L K^{\prime}\right\rangle .
$$

Since $|G\rangle$ differs from $\left|(\lambda \mu) \epsilon \Lambda M_{\Lambda}\right\rangle$ by at most an $n_{i}$ dependent phase factor and $n_{i}^{(1)}+n_{i}^{(2)}=n_{i}^{(3)}$, it is convenient to write

$$
\begin{aligned}
& \left\langle G_{1} ; G_{2} \mid G_{3}\right\rangle_{\rho} \\
& =\left\langle\left(\lambda_{1} \mu_{1}\right) \epsilon_{1} \Lambda_{1} M_{\Lambda_{1}} ;\left(\lambda_{2} \mu_{2}\right) \epsilon_{2} \Lambda_{2} M_{\Lambda_{2}} \mid\left(\lambda_{3} \mu_{3}\right) \epsilon_{3} \Lambda_{3} M_{\Lambda_{3}}\right\rangle_{\rho} .
\end{aligned}
$$

The $S_{3} \supset R_{3}$ Wigner coefficients are then given by

$$
\begin{aligned}
\left\langle\left(G_{1 E}\right) \mathcal{K}_{1} L_{1} M_{1} ;\left(G_{2 E}\right) \mathcal{K}_{2} L_{2} M_{2} \mid\left(G_{3 E}\right) \mathcal{K}_{3} L_{3} M_{3}\right\rangle_{\rho} \\
=\sum_{\delta_{1} \mathcal{E}_{2} \varepsilon_{3}}\left\langle G_{1} \mid\left(G_{1 E}\right) \mathcal{K}_{1} L_{1} M_{1}\right\rangle\left\langle G_{2} \mid\left(G_{2 E}\right) \mathcal{K}_{2} L_{2} M_{2}\right\rangle \\
\quad \times\left\langle G_{3} \mid\left(G_{3 E}\right) \mathcal{K}_{3} L_{3} M_{3}\right\rangle\left\langle G_{1} ; G_{2} \mid G_{3}\right\rangle_{\rho} .
\end{aligned}
$$

An expression which is more convenient to evaluate from a computational point of view may be obtained by directly expanding the inner product

$$
\begin{aligned}
\left\langle\left(G_{1 E}\right)\right. & \mathcal{K}_{1} L_{1} M_{1} ;\left(G_{2 E}\right) \mathcal{K}_{2} L_{2} M_{2}\left|\left(G_{3 E}\right) K_{3} L_{3} M_{3}\right\rangle_{\rho} \\
& =\left\langle\left(G_{1 E}\right) \mathcal{K}_{1} L_{1} M_{1} ;\left(G_{2 E}\right) \mathcal{K}_{2} L_{2} M_{2}\left|P_{M_{3} K_{3}}^{L_{3}}\right| G_{3 E}\right\rangle_{\rho} .
\end{aligned}
$$

Making use of the fact that $R_{3}(\Omega)=R_{1}(\Omega) R_{2}(\Omega)$, the effect of the projection operator acting to the left can be determined. Integrating over Euler angles by means of the Clebsch-Gordan series for rotation matrices then leads to the result

$$
\begin{aligned}
& \left\langle\left(G_{1 E}\right) \mathcal{K}_{1} L_{1} M_{1} ;\left(G_{2 E}\right) \mathcal{K}_{2} L_{2} M_{2} \mid\left(G_{3 E}\right) K_{3} L_{3} M_{3}\right\rangle_{\rho} \\
& =\sum_{\substack{\boldsymbol{g}_{1} \xi_{2} \xi_{2} \\
M_{1}^{\prime}\left(M_{2}^{\prime}\right)}}\left\langle L_{1} M_{1}^{\prime} ; L_{2} M_{2}^{\prime} \mid L_{3} K_{3}\right\rangle\left\langle G_{1} \mid\left(G_{1 E}\right) \mathcal{K}_{1} L_{1} M_{1}^{\prime}\right\rangle \\
& \quad \times\left\langle G_{2} \mid\left(G_{2 E}\right) \mathcal{K}_{2} L_{2} M_{2}^{\prime}\right\rangle\left\langle G_{1} ; G_{2} \mid G_{3 E}\right\rangle_{\rho} \\
& \quad \times\left\langle L_{1} M_{1} ; L_{2} M_{2} \mid L_{3} M_{3}\right\rangle .
\end{aligned}
$$

Applying Eq. (5) to the 3-space yields the required $S U_{3} \supset$ $R_{3}$ Wigner coefficients. Note that the summation in this case is only over $S U_{3} \supset S U_{2} \times U_{1}$ Wigner coefficients of the type $G_{3}=G_{3 E}$, i.e., those which can be evaluated through Eqs. (17)-(18) without the use of Eq. (19). Clearly a factorization into the product of a reduced $\mathrm{SU}_{3} \supset R_{3}$ Wigner coefficient and an ordinary Wigner coefficient in $R_{3}$ space is possible. Note that it is unnecessary and indeed redundant to fix the phase for the $S U_{3} \supset R_{3}$ Wigner coefficients independently of that already chosen for the $S U_{3} \supset S U_{2} \times U_{1}$ reduction. The orthonormality of the transformation coefficients between the two schemes guarantees a unique solution. In effect the choice is made by selecting positive roots in Eq. (6).

\section{CONJUGATION AND SYMMETRY PROPERTIES}

Since the $S U_{3} \supset R_{3}$ reduction is linked to the $S U_{3} \supset$ $S U_{2} \times U_{1}$ reduction via the transformation coefficients of Eq. (24), it suffices to make a determination of the conjugation relationship and all symmetry properties for the $S U_{3} \supset S U_{2} \times U_{1}$ reduction only. The corresponding $S U_{3} \supset R_{3}$ results follow from known relationships among the transformation coefficients between the two schemes.

\section{A. State conjugation}

The transformation coefficients $\left\langle G \mid\left(G_{E}\right) \mathcal{K} L M\right\rangle$ are the elements of a real unitary (hence orthogonal) matrix if $|G\rangle=(i)^{n_{1}+n_{3}}\left|\left(h_{i j}\right)\right\rangle$, where the $\left|\left(h_{i j}\right)\right\rangle$ are states of the type defined by Moshinsky in terms of polynomials in creation operators acting on the vacuum. ${ }^{24}$ [The choice $(i)^{n_{2}}\left|\left(h_{i j}\right)\right\rangle$ as suggested in Ref. 23 is also acceptable. In this case, however, the states would not transform according to conventional phasing under the $R T$ operation ( $\pi$-rotation about the 2 axis $\times$ time reversal) as defined by Bohr and Mottelson. ${ }^{25}$ ] The results given in Appendix A2 of Ref. 19 for the adjoint irreducible representation can then be used to show that 26

$$
\left.\begin{array}{l}
|G\rangle^{*}=(-1)^{p-r}|\bar{G}\rangle, \\
\tilde{\lambda}=\mu, \quad \tilde{\mu}=\lambda, \\
\tilde{p}=\mu-q \\
\tilde{q}=\lambda-p \\
\tilde{r}=p+\mu-q-r
\end{array}\right\} \Rightarrow \begin{gathered}
\tilde{\epsilon}=-\epsilon \\
\tilde{\Lambda}=\Lambda \\
\tilde{M}_{\Lambda}=-M_{\Lambda}
\end{gathered}
$$

Note that $p-r=\frac{1}{3}(\lambda-\mu)-\frac{1}{8} \epsilon-M_{\Lambda}=\tilde{r}-\tilde{p}$. The sign of $M_{\Lambda}$ differs from that of Hecht due to the choice $M_{\Lambda}=$ $r-\Lambda$ of Eq. (1). [This choice allows the more natural correspondence $(z x y) \sim(312)$ rather than $(z x y) \sim(321)$ to be made between body-fixed axes $x, y, z$ and the $i, j$ labels of the Gel'fand scheme.] For $G=G_{E}$ Eq. (32) implies that in addition to $\lambda$ and $\mu$ interchanging roles $I=$ $1-I$ and $\widetilde{J}=1-J$, where $I$ and $J$ are as defined by Table I; that is, under conjugation $\mathrm{HW} \rightarrow \mathrm{LW}$ and $\mathrm{LW} \rightarrow \mathrm{HW}$.

To discover the conjugation properties of the $\left|\left(G_{E}\right) \pi L M\right\rangle$ it suffices to know in addition to Eq. (32) the symmetry properties of the $\left\langle G \mid\left(G_{E}\right) K L M\right\rangle$. By straightforward but tedious substitution it can be shown that for the inner product of $\left|G^{\prime}\right\rangle$ with a state $\left|(G) M L M^{\prime}\right\rangle$ [defined by Eq. (3)],

$$
\begin{aligned}
& \text { 1. }\left\langle G^{\prime} \mid(G) M L M^{\prime}\right\rangle^{*}=\left\langle G^{\prime} \mid(G) M L M^{\prime}\right\rangle, \\
& \text { 2. }\left\langle G^{\prime} \mid(G)-M, L,-M^{\prime}\right\rangle \\
& =(-1)^{n_{2}-n_{2}^{\prime}+M-M^{\prime}}\left\langle G^{\prime} \mid(G) M L M^{\prime}\right\rangle,
\end{aligned}
$$

3. $\left\langle G^{\prime} \mid(G) M L M^{\prime}\right\rangle=(-1)^{n_{3}-n_{3}^{\prime}+M-M^{\prime}}\left\langle G \mid\left(G^{\prime}\right) M^{\prime} L M\right\rangle$,

4. $\left\langle\tilde{G}^{\prime} \mid(\tilde{G}) M L M^{\prime}\right\rangle=\left\langle G^{\prime} \mid(G) M L M^{\prime}\right\rangle$,

5A. $\left\langle G^{\prime} \mid(G) M L,-M^{\prime}\right\rangle=(-1)^{n_{1}^{\prime}-n_{3}+L+M}\left\langle G^{\prime} \mid(G) M L M^{\prime}\right\rangle$,

5B. $\left\langle G^{\prime} \mid(G)-M L M^{\prime}\right\rangle=(-1)^{n_{1}-n_{3}^{\prime}+L+M^{\prime}}\left\langle G^{\prime} \mid(G) M L M^{\prime}\right\rangle$, 6A. $\left\langle G^{\prime}\left(M_{\Lambda}^{\prime}\right) \mid(G) M L M^{\prime}\right\rangle=(-1)^{\Lambda^{\prime}+M^{\prime} / 2}\left\langle G^{\prime}\left(-M_{\Lambda}^{\prime}\right) \mid(G) M L M^{\prime}\right\rangle$, 6B. $\left\langle G^{\prime} \mid\left(G\left(M_{\Lambda}\right)\right) M L M^{\prime}\right\rangle=(-1)^{\Lambda+M / 2}\left\langle G^{\prime} \mid\left(G\left(-M_{\Lambda}\right)\right) M L M^{\prime}\right\rangle$.

Since $\nVdash=K+2 n$ where $n$ is integral, the symmetries apply directly to the $\left\langle G \mid\left(G_{E}\right) \mathcal{K} L M\right\rangle$ as well as the $\left\langle G \mid\left(G_{E}\right) K L M\right\rangle$. Property 6 together with property 1 insures that the $\left\langle G^{\prime} \mid(G) M L M^{\prime}\right\rangle$ vanish for either $2 \Lambda^{\prime}+M^{\prime}$ or $2 \Lambda+M$ odd. Properties $1,5 \mathrm{~A}, 4$ can then be used to show that

$$
\left|\left(G_{E}\right) \mathcal{K} L M\right\rangle^{*}=(-1)^{\lambda+\mu+L-M}\left|\left(G_{E}\right) \mathcal{K} L,-M\right\rangle .
$$

Note that $G_{E}=G_{\mathrm{HW}}\left(G_{\mathrm{LW}}\right)$ implies Eq. (4a) [Eq. (4b)] applies on the left whereas Eq. (4b) [Eq. (4a)] applies on the right. But since $\lambda$ and $\mu$ also interchange roles, $\mathcal{K}$ is left invariant.

\section{B. Symmetry properties}

In Sec. 3 a prescription is given for a unique determination, including phase, of all $S U_{3} \supset S U_{2} \times U_{1}$ Wigner coefficients. In terms of $\varphi=\lambda_{1}+\lambda_{2}-\lambda_{3}+\mu_{1}+\mu_{2}-\mu_{3}$
which is even or odd as $\left(\lambda_{1}+\lambda_{2}-\lambda_{3}-\mu_{1}-\mu_{2}+\mu_{3}\right) / 3$ $=p_{1}-r_{1}+p_{2}-r_{2}-p_{3}+r_{3}$ is even or odd, the corresponding symmetry properties are:

Symmetry Properties of the $S U_{3} \supset S U_{2} \times U_{1}$ Wigner Coefficients 


$$
\begin{aligned}
& \text { 1A. }\left\langle G_{1} ; G_{2} \mid G_{3}\right\rangle_{\rho} \\
& =(-1)^{\varphi+p_{2}-r_{2}} \sqrt{\operatorname{dim}\left(\lambda_{3} \mu_{3}\right) / \operatorname{dim}\left(\lambda_{1} \mu_{1}\right)}\left\langle G_{3} ; \tilde{G}_{2} \mid G_{1}\right\rangle_{\rho}, \\
& \text { 2A. }\left\langle G_{1} ; G_{2} \mid G_{3}\right\rangle_{\rho}=(-1)^{\varphi+\eta_{\max }-\rho}\left\langle G_{1} ; \tilde{G}_{2} \mid \tilde{G}_{3}\right\rangle_{\rho}, \\
& \text { 3A. }\left\langle G_{1} ; G_{2} \mid G_{3}\right\rangle=(-1)^{\varphi}\left\langle G_{2} ; G_{1} \mid G_{3}\right\rangle \quad\left(\eta_{\max }=1 \text { only }\right), \\
& \text { 1B. }\left\langle G_{1} ; G_{2} \| G_{3}\right\rangle_{\rho} \\
& =(-1)^{\varphi+\frac{1}{3}\left(\lambda_{2}-\mu_{2}\right)-\frac{1}{6} \epsilon_{2}+\Lambda_{3}-\Lambda_{1}} \\
& \quad \times \sqrt{\operatorname{dim}\left(\lambda_{3} \mu_{3}\right)\left(2 \Lambda_{1}+1\right) / \operatorname{dim}\left(\lambda_{1} \mu_{1}\right)\left(2 \Lambda_{3}+1\right)} \\
& \quad \times\left\langle G_{3} ; \tilde{G}_{2} \| G_{3}\right\rangle_{\rho}, \\
& \text { 2B. }\left\langle G_{1} ; G_{2} \| G_{3}\right\rangle_{\rho}=(-1)^{\varphi+\eta_{\max }-\rho+\Lambda_{1}+\Lambda_{2}-\Lambda_{3}}\left\langle\tilde{G}_{1} ; \tilde{G}_{2} \| \tilde{G}_{3}\right\rangle_{\rho}, \\
& \text { 3B. }\left\langle G_{1} ; G_{2} \| G_{3}\right\rangle_{\rho}=(-1)^{\varphi+\Lambda_{2}+\Lambda_{2}-\Lambda_{3}}\left\langle G_{2} ; G_{1} \| G_{3}\right\rangle \\
& \quad\left(\eta_{\max }=1\right. \text { only). }
\end{aligned}
$$

Among these, the most important is Symmetry 1. Expression (20) satisfies this relation, from which it follows that it holds for the coefficients $\left\langle G_{1} ; \bar{G}_{2} \mid G_{3}\right\rangle_{\rho}$ and $\left\langle G_{3} ; \bar{G}_{2} \mid G_{1}\right\rangle_{\rho}$. A comparison of the expression for $\left\langle G_{1} ; G_{2} \mid G_{3}\right\rangle_{\rho}$ given by the right-hand side of Eq. (13) with that for $\left\langle G_{3} ; G_{2} \mid G_{1}\right\rangle_{\rho}$ given by the right-hand side of Eq. (13') then sufficies by induction to establish the relationship for the general case. The validity of Symmetry 2, apart from phase, is a direct consequence of the symmetric nature of the formulation under the operation of conjugation. The appearance of the phase factor in this case, however, is by no means obvious. The factor $(-1)^{\varphi}$ is a direct consequence of Eq. (32). But, as already suggested, 26 consistency requires an additional phase, $\xi= \pm 1$. It has been determined that $\left\langle G_{1} ; \bar{G}_{2} \mid G_{3}\right\rangle_{\rho}=$ $(-1) \varphi\left\langle\dot{G}_{1} ; \overline{\bar{G}}_{2} \mid G_{3}\right\rangle_{\rho}$, i.e., $\xi=+1$ for this special variety. The general result, $\xi=(-1)^{\eta} \max ^{-\rho}$, then follows from recursion relation (13). An arbitrary resolution of the multiplicity would, in general, require a linear transformation among the $\rho$-labels on the right-hand side of each of Eqs. (35). The significance of the "canonical" decomposition manifests itself in Symmetry 1 and Symmetry 2, where such a transformation does not appear and the multiplicity label $\rho$ is the same on both sides of the equations. This, however, is not the case for Symmetry 3 because of the unsymmetric treatment of $G_{1}$ and $G_{2}$ and accounts for the restriction $\eta_{\max }=1$, i.e., multiplicity free couplings only.

Practical considerations may favor adopting a different phase convention. ${ }^{21}$ But doing so requires a modification in the phases for the symmetries of Eq. (35). For example, under the convention adopted by Hecht, namely requiring $\left\langle\left(\lambda_{1} \mu_{1}\right) \mathrm{LW} ;\left(\lambda_{2} \mu_{2}\right) \epsilon_{2} \Lambda_{2} \|\left(\lambda_{3} \mu_{3}\right) L W\right\rangle_{p}>0$, the results can be summarized as follows:

Symmetry 1 remains unchanged, Symmetry 2 holds with $\eta_{\max }$ replaced by $\rho_{\max }$, Symmetry 3 is valid for $\rho_{\max }=1$ only. That is, in this particularly simple case all that is required is for $\eta_{\max }$ to be replaced by $\rho_{\max }$ throughout.

The symmetry properties of the $S U_{3} \supset R_{3}$ Wigner coefficients can be obtained from those given above by using the results of Eqs. (33) together with Eq. (34).

Symmetry Properties of the $\mathrm{SU}_{3} \supset R_{3}$ Wigner Coefficients

$$
\begin{aligned}
& \text { 1A. }\left\langle\left(G_{1 E}\right) \mathcal{K}_{1} \Sigma_{1} M_{1} ;\left(G_{2 E}\right) \mathcal{K}_{2} L_{2} M_{2} \mid\left(G_{3 E}\right) \mathcal{K}_{3} L_{3} M_{3}\right\rangle_{\rho} \\
& =(-1)^{\varphi+\lambda_{2}+\mu_{2}+L_{2}+M_{2}} \sqrt{\operatorname{dim}\left(\lambda_{3} \mu_{3}\right) / \operatorname{dim}\left(\lambda_{1} \mu_{1}\right)} \\
& \times\left\langle\left(G_{3 E}\right) \mathcal{K}_{3} L_{3} M_{3} ;\left(\widetilde{G}_{2 E}\right) \mathcal{K}_{2} L_{2},-M_{2} \mid\left(G_{3 E}\right) \mathcal{K}_{3} L_{3} M_{3}\right\rangle_{\rho} \\
& \text { 2A. }\left\langle\left(G_{1 E}\right) \mathcal{K}_{1} L_{1} M_{1} ;\left(G_{2 E}\right) K_{2} L_{2} M_{2} \mid\left(G_{3 E}\right) K_{3} L_{3} M_{3}\right\rangle_{\rho} \\
& =(-1)^{\varphi+\eta_{\max }-\rho+L_{1}+L_{2}-L_{3}} \\
& \times\left\langle\left(\tilde{G}_{1 E}\right) \mathcal{K}_{1} L_{1},-M_{1} ;\left(\tilde{G}_{2 E}\right) \mathcal{K}_{2} L_{2},-M_{2} \mid\left(\tilde{G}_{3 E}\right) \mathcal{K}_{3} L_{3},-M_{3}\right\rangle_{p},
\end{aligned}
$$

3A. $\left\langle\left(G_{1 E}\right) \mathcal{K}_{1} L_{1} M_{1} ;\left(G_{2 E}\right) \mathcal{K}_{2} L_{2} M_{2} \mid\left(G_{3 E}\right) \mathcal{K}_{3} L_{3} M_{3}\right\rangle=(-1)^{\varphi}$

$$
\begin{array}{r}
\times\left\langle\left(G_{2 E}\right) \mathcal{K}_{2} L_{2} M_{2} ;\left(G_{1 E}\right) \mathcal{K}_{1} L_{1} M_{1} \mid\left(G_{3 E}\right) \mathcal{K}_{3} L_{3} M_{3}\right\rangle \\
\left(\eta_{\max }=1 \text { only }\right),
\end{array}
$$

1B. $\left\langle\left(G_{1 E}\right) \mathfrak{K}_{1} L_{1} ;\left(G_{2 E}\right) \mathfrak{K}_{2} L_{2} \|\left(G_{3 E}\right) \mathfrak{K}_{3} L_{3}\right\rangle_{p}$

$$
\begin{aligned}
= & (-1)^{\varphi+\lambda_{2}+\mu_{2}+L_{1}+L_{2}-L_{3}} \\
& \times \sqrt{\operatorname{dim}\left(\lambda_{3} \mu_{3}\right)\left(2 L_{1}+1\right) / \operatorname{dim}\left(\lambda_{1} \mu_{1}\right)\left(2 L_{3}+1\right)} \\
& \left\langle\left(G_{3 E}\right) \mathcal{K}_{3} L_{3} ;\left(G_{2 E}\right) \mathcal{K}_{2} L_{2} \|\left(G_{1 E}\right) \mathcal{K}_{1} L_{1}\right\rangle_{\rho},
\end{aligned}
$$

2B. $\left\langle\left(G_{1 E}\right) \mathcal{K}_{1} L_{1} ;\left(G_{2 I}\right) \hat{q}_{2} L_{2} \|\left(G_{3 E}\right) \mathcal{K}_{3} L_{3}\right\rangle_{\rho}=(-1)^{\varphi+\eta_{\max }-\rho}$

$$
\times\left\langle\left(\tilde{G}_{1 E}\right) \mathcal{K}_{1} L_{1} ;\left(\tilde{G}_{2 E}\right) \mathcal{K}_{2} L_{2} \|\left(\tilde{G}_{3 E}\right) \mathcal{K}_{3} L_{3}\right\rangle_{\rho},
$$

3B. $\left\langle\left(G_{1 E}\right) \mathcal{K}_{1} L_{1} ;\left(G_{2 E}\right) \mathfrak{K}_{2} L_{2} \|\left(G_{3 E}\right) \mathfrak{K}_{3} L_{3}\right\rangle=(-1)^{\varphi+L_{1}+L_{2}-L_{3}}$

$$
\begin{array}{r}
\times\left\langle\left(G_{2 E}\right) \mathcal{K}_{2} L_{2} ;\left(G_{1 E}\right) \mathcal{K}_{1} L_{1} \|\left(G_{3 E}\right) \mathcal{K}_{3} L_{3}\right\rangle \\
\left(\eta_{\max }=1 \text { only }\right) .
\end{array}
$$

Again, under the convention of Hecht, these relations hold if $\eta_{\max }$ is replaced by $\rho_{\max }$ throughout.

\section{CONCLUDING REMARKS}

The techniques described above developed as an outgrowth of the need for an advanced $S U_{3}$ technology in shell model calculations for light nuclei assuming general two-body effective interactions. ${ }^{27}$ Machine codes based on the results are therefore available. ${ }^{28}$ They allow a numerical determination of $S U_{3} \supset S U_{2} \times U_{1}$ and $S U_{3}$ $\supset R_{3}$ Wigner coefficients as well as $S U_{3}^{2}$ Racah coefficients to be made for arbitrary couplings and multiplicity.

Although the emphasis in the present article has been on the practical aspects of calculating $S U_{3}$ Wigner and Racah coefficients, it is quite possible, and indeed likely, that the build-up process using the group generators can be applied to the $\Gamma_{s}$ Wigner operators of Biedenharn and Louck and co-workers for the couplings $\left(\lambda_{1} \mu_{1}\right) \times\left(\bar{\lambda}_{2} \bar{\mu}_{2}\right)$ $\rightarrow\left(\lambda_{3} \mu_{3}\right), \rho=1,2, \ldots, \rho_{\max }$ to obtain the full set of Wigner operators for the coupling $\left(\lambda_{1} \mu_{1}\right) \times\left(\lambda_{2} \mu_{2}\right) \rightarrow$ $\left(\lambda_{3} \mu_{3}\right)$. Because of nonorthogonality, however, it is not clear that a simple interpretation of the structure of the operators in terms of geometrical properties of the socalled arrow patterns will be possible. Nevertheless, since our purpose in the present article is to avoid the luxury of mathematical sophistication the validity of such conjectures must be relegated to a later work.

\section{ACKNOWLEDGMENTS}

Thanks are due to nearly all the individuals with whom the authors had contact during the period in which the results reported in this article developed from mere speculation to concrete formulation. Special thanks, however, are due to $\mathrm{K}$. T. Hecht for the expertise he provided in this technical phase of our research into the structure of light nuclei. It is also a pleasure to acknowledge the support of W.C. Parkinson and the cyclotron group at Michigan.

*Work supported by the U.S. National Science Foundation

$\uparrow$ Present address: Department of Physics, University of Rochester, Rochester, New York.

¥ Present address: Department of Physics, Nihon University, Setagaya, Tokyo, Japan.

${ }^{1}$ E. P. Wigner, Group Theory and Its Application to the Quantum Mechanics of Atomic Spectra, translated from the German by J. J. Griffin (Academic, New York, 1959). 
${ }^{2}$ G. Racah, "Theory of Complex Spectra," in Quantum Mechanics of Angular Momentum, edited by L. C. Biedenharn and H. Van Dam (Academic; New York, 1965).

3J. P. Elliott, Proc. R. Soc. A 245, 128, 562 (1958).

${ }^{4}$ Y. Ne'eman, Nucl. Phys. 26, 222 (1961). M. Gell-Mann, Phys. Rev. 125, 1067 (1962).

${ }^{5}$ See, for example, references cited by C. K. Chew and R. T. Sharp, Nucl. Phys. B 2, 697 (1967).

${ }^{6}$ L. C. Biedenharn and J. D. Louck, J. Math. Phys. 13, 1985 (1972).

${ }^{7}$ L. C. Biedenhard, J. D. Louck, E. Chacón, and M. Ciftan, J. Math. Phys. 13, 1957 (1972).

${ }^{8}$ E. Chacón, M. Ciftan, and L. C. Biedenharn, J. Math. Phys. 13, 577 (1972).

${ }^{9}$ J. D. Louck and L. C. Biedenharn, J. Math. Phys. 12, 173 (1971).

${ }^{10} \mathrm{~J}$. D. Louck and L. C. Biedenharn, J. Math. Phys. 11, 2368 (1970).

${ }^{11}$ J. A. Castilho Alcarás, L. C. Biedenharn, K. T. Hecht, and G. Neeley, Ann. Phys. (N.Y.) 60, 85 (1970).

${ }^{12}$ J. D. Louck, Am. J. Phys. 38, 3 (1970) presents a bibliography and synthesis of published results on the theory of tensor operators in the unitary groups.

${ }^{13}$ J. P. Elliott and M. Harvey, Proc. R. Soc. A 272, 557 (1962).

${ }^{14}$ R. E. Behrends, J. Dreitlin, C. Fronsdal, and B. W. Lee, Rev. Mod.

Phys. 34, 1 (1962). J. J. deSwart, Rev. Mod. Phys. 35, 916 (1963).

${ }^{15}$ I. M. Gel'fand and M. L. Zeitlin, Dokl. Akad. Nauk SSSR 71, 825 (1950).

${ }^{16}$ G. Racah, "Group Theory and Spectroscopy" (Lecture Notes, Princeton, 1951). V. Bargmann and M. Moshinsky, Nucl. Phys. 18, 697 (1960); Nucl. Phys. 23, 177 (1961). R. T. Sharp and Hans C. Von Baeyer, Nucl. Phys. A. 127, 513 (1969).
${ }^{17} \mathrm{M}$. Harvey, in Advances in Nuclear Physics, edited by M. Baranger and and E. Vogt (Plenum, New York, 1971), Vol. 1.

${ }^{18}$ J. D. Vergados, Nucl. Phys. A 111, 681 (1968).

${ }^{19}$ K. T. Hecht, Nucl. Phys. 62, 1 (1965).

${ }^{20}$ A. R. Edmonds, Angular Momentum in Quantum Mechanics (Princeton U. P., Princeton, N. J., 1957).

${ }^{21}$ In addition to Refs. 14 and 19 see, for example, M. Moshinsky, Rev. Mod. Phys. 34, 813 (1962). A. R. Edmonds, Proc. R. Soc. A 268, 567 (1963).

${ }^{22}$ A. deShalit and I. Talmi, Nuclear Shell Theory (Academic, New York, 1963).

${ }^{23}$ J. P. Draayer and S. A. Williams, Nucl. Phys. A 129, 647 (1969).

${ }^{24}$ E. Chacón and M. Moshinsky, Phys. Lett. 23, 567 (1966); in Spectroscopic and Group Theoretical Concepts and Methods in Elementary Particle Physics, edited by F. Bloch, S. G. Cohen, A. deShalit, S. Sambursky, and I. Talmi (North-Holland, Amsterdam, 1968).

${ }^{25}$ A. Bohr and Ben R. Mottelson, Nuclear Structure (Benjamin, New York, 1969).

${ }^{26}$ For a coupled system $\left(\lambda_{1} \mu_{1}\right) \times\left(\lambda_{2} \mu_{2}\right) \rightarrow\left(\lambda_{3} \mu_{3}\right)$, Eq. (32) cannot simultaneously be applied to all three representations $\left(\lambda_{1} \mu_{1}\right),\left(\lambda_{2} \mu_{2}\right)$, and $\left(\lambda_{3} \mu_{3}\right)$ in a consistent fashion. An additional dependence upon the multiplicity label is required. See, for example, J. J. deSwart, Rev. Mod. Phys. 35, 916 (1963), Sec. 14 and Sec. 4B of the current article.

${ }^{27}$ J. P. Draayer, Bull. Am. Phys. Soc. 18 (1), 137 (1973). R. D. Ratna Raju, J. P. Draayer, and K. T. Hecht, Nucl. Phys. A 202, 433 (1973).

${ }^{28}$ J. P. Draayer and Yoshimi Akiyama, Comput. Phys. Commun. 5, 405 (1973). 